TRANSACTIONS OF THE

AMERICAN MATHEMATICAL SOCIETY

Volume 362, Number 11, November 2010, Pages 5909-5946

S 0002-9947(2010)04932-3

Article electronically published on June 2, 2010

\title{
A SOLVABLE VERSION OF THE BAER-SUZUKI THEOREM
}

\author{
SIMON GUEST
}

\begin{abstract}
Suppose that $G$ is a finite group and $x \in G$ has prime order $p \geq 5$. Then $x$ is contained in the solvable radical of $G, O_{\infty}(G)$, if (and only if) $\left\langle x, x^{g}\right\rangle$ is solvable for all $g \in G$. If $G$ is an almost simple group and $x \in G$ has prime order $p \geq 5$, then this implies that there exists $g \in G$ such that $\left\langle x, x^{g}\right\rangle$ is not solvable. In fact, this is also true when $p=3$ with very few exceptions, which are described explicitly.
\end{abstract}

\section{INTRODUCTION}

The Baer-Suzuki theorem provides a useful characterization of the Fitting subgroup of a finite (or linear) group. It can be stated as follows:

Theorem 1 (Baer-Suzuki). Let $G$ be a finite (or linear) group. Suppose that for some $x \in G,\left\langle x, x^{g}\right\rangle$ is nilpotent for all $g \in G$. Then $\left\langle x^{G}\right\rangle$ is nilpotent. That is, $x$ is contained in the Fitting subgroup of $G$.

It is natural to ask if there is an analogous result if the nilpotency condition is replaced with solvability. However, it is easy to find counterexamples. For example, any two involutions generate a dihedral group. So if $G$ is a non-abelian simple group and $x$ is an involution in $G$, then $\left\langle x^{G}\right\rangle=G$ is not solvable yet $\left\langle x, x^{g}\right\rangle$ is solvable for all $g \in G$.

There are also counterexamples when $x$ has order 3 . Suppose that $x \in S L(n, 3)$ $(n \geq 3)$ has order 3 and acts trivially on some hyperplane; that is, $x$ is a transvection. Then $x$ and any conjugate $x^{g}$ generate a group that acts trivially on a subspace of codimension at most 2. Thus $\left\langle x, x^{g}\right\rangle$ is solvable since it has a normal abelian subgroup $N$ such that $\left\langle x, x^{g}\right\rangle / N$ is isomorphic to a subgroup of $G L(2,3)$. However, since $x$ is not central, it is not contained in the solvable radical of $S L(n, 3)$ and $\left\langle x^{G}\right\rangle$ is not solvable. The aim is to prove the following:

Theorem A. Let $G$ be a finite group. Suppose that $x \in G$ has prime order $p \geq 5$. If $\left\langle x, x^{g}\right\rangle$ is solvable for all $g \in G$, then $\left\langle x^{G}\right\rangle$ is solvable. Equivalently, if $x \notin O_{\infty}(G)$, then there exists $g \in G$ such that $\left\langle x, x^{g}\right\rangle$ is not solvable.

Received by the editors January 25, 2008 and, in revised form, September 14, 2008.

2000 Mathematics Subject Classification. Primary 20F14, 20D10.

The author was partially supported by the NSF grant DMS 0653873.

(C)2010 American Mathematical Society Reverts to public domain 28 years from publication 
It is worth noting that Theorem A implies the following result:

Corollary 1. Let $G$ be a finite (or linear) group. Then $G$ is solvable if and only if any two conjugates generate a solvable group.

Proof. Let $G$ be a minimal counterexample to the version of the theorem for finite groups. Thus $G$ is a finite simple group by minimality, and therefore $G$ contains an element $x$ of prime order $p \geq 5$. So Theorem A implies that there exists $g \in G$ such that $\left\langle x, x^{g}\right\rangle$ is not solvable, and thus $G$ is not a minimal counterexample. The version of the theorem for linear groups follows from the finite group version using a standard argument (see [FGG, Corollary 1.2] for example).

Also note that a minimal counterexample in Theorem 1 must be one of the minimal simple groups described by Thompson in the $N$-group paper [Tho68. Thus one could prove Theorem 1 without relying on the full Classification theorem by ruling out all of the minimal simple groups.

Theorem A is also used in FGG to prove:

Theorem 2. Let $G$ be a finite or linear group. Then $x \in G$ is contained in the solvable radical of $G$ if and only if $\left\langle x, x^{g_{1}}, x^{g_{2}}, x^{g_{3}}\right\rangle$ is solvable for all $g_{1}, g_{2}, g_{3} \in G$.

The proof in [FGG] relies on the Classification of Finite Simple Groups; however, a weaker version of the theorem for finite groups is also given in [FGG] that does not rely on the Classification theorem:

Theorem 3. Let $G$ be a finite group. Then $x \in G$ is contained in the solvable radical of $G$ if and only if every 7 conjugates of $x$ generate a solvable group.

Theorems A and 2 were announced in GPS07] (see Theorems 7.3 and 7.4). They have been obtained independently in GGKP08b] and [GGKP08a], also using the Classification theorem.

\section{REDUCTION}

Lemma 1 below simplifies matters considerably. It reduces the proof to a situation where $G$ is an almost simple group.

Lemma 1. Suppose that $G$ is a finite group such that the Fitting subgroup $F(G)$ is trivial. Let $L$ be a component of $G$.

(a) If $x$ is an element of $G$ such that $x \notin N_{G}(L)$ and $x^{2} \notin C_{G}(L)$, then there exists an element $g$ in $G$ such that $\left\langle x, x^{g}\right\rangle$ is not solvable.

(b) If $x$ is an element of $G$ such that $x \notin N_{G}(L)$ and $x^{2} \in C_{G}(L)$, then there exist elements $g_{1}$ and $g_{2}$ in $G$ such that $\left\langle x, x^{g_{1}}, x^{g_{2}}\right\rangle$ is not solvable.

Proof. Write $E(G)$ for the subgroup of $G$ generated by its components. Then the generalized Fitting subgroup is $F^{*}(G)=E(G) F(G)$. Since $F(G)=1$, it follows that $Z\left(F^{*}(G)\right)=Z(E(G))$ is a normal abelian subgroup of $G$ and is therefore trivial. Also, $Z(E(G))$ is generated by the centers of each component of $G$ and so all of the components of $G$ are simple. Moreover, $E(G)$ must be a direct product of the components of $G$. So $G$ is embedded in $\operatorname{Aut}\left(F^{*}(G)\right)=\operatorname{Aut}(E(G))$. It suffices to assume that $G=\langle L, x\rangle$. Thus if $t:=\mid\left\{L^{x^{i}}:\right.$ for $\left.i=1,2, \ldots\right\} \mid$, then $E(G)=L \times \cdots \times L^{x^{t-1}}$ and $\operatorname{Aut}(E(G)) \cong \operatorname{Aut}(L) \imath S_{t}$. Since $x$ does not normalize 
$L$, it follows that $t \geq 2$. Moreover, it suffices to assume that $x=\left(\sigma_{1}, \ldots, \sigma_{t}\right) \tau$, where $\sigma_{i} \in \operatorname{Aut}\left(L^{x^{i-1}}\right)$ and $\tau$ is the $t$-cycle $(12 \cdots t)$. Now observe that

$$
\begin{aligned}
x^{\left(u_{1}, \ldots, u_{t}\right)} & =\left(u_{1}, \ldots, u_{t}\right)\left(\sigma_{1}, \ldots, \sigma_{t}\right) \tau\left(u_{1}, \ldots, u_{t}\right)^{-1} \tau^{-1} \tau \\
& =\left(u_{1}, \ldots, u_{t}\right)\left(\sigma_{1}, \ldots, \sigma_{t}\right)\left(u_{t}^{-1}, u_{1}^{-1}, \ldots, u_{t-1}^{-1}\right) \tau .
\end{aligned}
$$

So if

$$
\begin{aligned}
& u_{t}=1, u_{t-1}=\sigma_{t}, u_{t-2}=\left(\sigma_{t} \sigma_{t-1}\right), u_{t-3}=\left(\sigma_{t} \sigma_{t-1} \sigma_{t-2}\right), \ldots, \\
& u_{1}=\left(\sigma_{t} \sigma_{t-1} \cdots \sigma_{1}\right)
\end{aligned}
$$

then $x^{\left(u_{1}, \ldots, u_{t}\right)}=(y, 1,1, \ldots, 1) \tau$ for some $y \in \operatorname{Aut}(L)$. Thus, it suffices to assume that $x$ is of this form.

Now let $g:=\left(w_{1}, \ldots, w_{t}\right) \in \operatorname{Aut}(L) \times \cdots \times \operatorname{Aut}\left(L^{x^{t-1}}\right)$ so that

$$
x^{-1}\left(w_{1}, \ldots, w_{t}\right) x\left(w_{1}^{-1}, \ldots, w_{t}^{-1}\right)=\left(w_{2} w_{1}^{-1}, \ldots\right)
$$

and

$$
\left(w_{1}, \ldots, w_{t}\right) x\left(w_{1}, \ldots, w_{t}\right)^{-1} x^{-1}=\left(w_{1} y w_{t}^{-1} y^{-1}, \ldots\right) .
$$

First, suppose that $t \geq 3$. By [AG84, Theorem B], there exist $l_{1}$ and $l_{2}$ in $L$ such that $L=\left\langle l_{1}, l_{2}\right\rangle$. So define $w_{1}=1, w_{2}=l_{1}$, and $w_{t}=y^{-1} l_{2} y$. Thus $\left\langle x, x^{g}\right\rangle$ contains $\left(l_{1}, \ldots\right)$ and $\left(l_{2}, \ldots\right)$ and is not solvable. If $t=2$ and $x^{2} \notin C_{G}(L)$, then $x=(y, 1) \tau$ and since $x^{2}=(y, y)$, it follows that $y \neq 1$. Now $\langle y, L\rangle$ is almost simple, so by GK00 there exists $z \in\langle y, L\rangle$ such that $\langle y, z\rangle$ contains $L$. Observe that there exists $l \in L$ such that $z=y^{k} l$. So define $w_{1}:=1$ and $w_{2}:=l$ and then

$$
\begin{aligned}
x^{2 k-1} x^{\left(w_{1}, w_{2}\right)} & =\left(y^{k}, y^{k-1}\right) \tau\left(w_{1}, w_{2}\right)(y, 1) \tau\left(w_{1}^{-1}, w_{2}^{-1}\right) \\
& =\left(y^{k} w_{2} w_{1}^{-1}, \cdot\right)=(z, \cdot)
\end{aligned}
$$

and so $\left\langle x, x^{\left(w_{1}, w_{2}\right)}\right\rangle$ cannot be solvable. This proves part (a).

To prove (b), suppose that $x$ does not normalize $L$ and $x^{2} \in C_{G}(L)$. So it suffices to assume that $t=2$ and $x=\tau$. If $g_{1}:=\left(1, l_{1}\right)$ and $g_{2}:=\left(1, l_{2}\right)$, then

$$
x^{-1} x^{g_{1}}=\left(l_{1}, \cdot\right) ; x^{-1} x^{g_{2}}=\left(l_{2}, \cdot\right)
$$

and thus $\left\langle x, x^{g_{1}}, x^{g_{2}}\right\rangle$ is not solvable. This proves part (b) of Lemma 1.

Lemma 2. Suppose that $(x, G)$ is a minimal counterexample. Then $G$ is almost simple.

Proof. Since $(x, G)$ is a minimal counterexample, the solvable radical of $G$ is trivial. Let $N$ be a minimal normal subgroup. So $N \cong L \times \cdots \times L$ for some non-abelian simple group $L$. If $x \in N$, then $G=N$ since otherwise $\left\langle x^{N}\right\rangle$ would be a solvable normal subgroup of $N$, and $N$ does not have any such subgroups. Thus, if $x \in N$, then $G$ is simple since $G$ has no non-trivial normal subgroups. Now assume that $x \notin N$ and let $H:=\langle x, N\rangle$. If $G \neq H$, then $\left\langle x^{H}\right\rangle \cap N$ is a solvable normal subgroup of $N$ and is thus trivial. Thus $[x, N]=1$, which is not possible, because it would follow that $\left[\left\langle x^{G}\right\rangle, N\right]=1$. Since $N$ is a minimal normal subgroup, $\left\langle x^{G}\right\rangle \cap N$ would be trivial and thus $\left\langle(x N)^{G / N}\right\rangle \cong\left\langle x^{G}\right\rangle N / N \cong\left\langle x^{G}\right\rangle$. This is not possible since $\left\langle(x N)^{G / N}\right\rangle$ is solvable by minimality. So $G=H=\langle x, N\rangle$. Note that the Fitting subgroup of $G$ is trivial since the solvable radical is trivial and thus $x$ normalizes 
TABLE 1. List of exceptions to Theorem A*

\begin{tabular}{|c|c|}
\hline$G_{0}$ & $x$ \\
\hline$P S L(n, 3), n>2$ & transvection \\
$P S p(2 n, 3), n>1$ & transvection \\
$P S U(n, 3), n>2$ & transvection \\
$P S U(n, 2), n>3$ & reflection of order 3 \\
$P \Omega^{\epsilon}(n, 3), n>6$ & $x$ a long root element \\
$E_{l}(3), F_{4}(3),{ }^{2} E_{6}(3),{ }^{3} D_{4}(3)$ & $x$ a long root element \\
$G_{2}(3)$ & $x$ a long or short root element \\
$G_{2}(2)^{\prime} \cong P S U(3,3)$ & transvection \\
\hline
\end{tabular}

every component by Lemma 1. So $L$ is normal in $G, N=L$ and $G=\langle x, L\rangle$. Now $G$ is almost simple since $L$ is the unique minimal normal subgroup of $G$.

The Classification of Finite Simple Groups can be used to determine the possibilities for the socle $G_{0}$ of $G$, and thus eliminate each possibility case by case. In fact, the following theorem is slightly stronger and implies Theorem A.

Theorem $\mathbf{A}^{*}$. Let $G$ be a finite almost simple group with socle $G_{0}$. Suppose that $x$ is an element of odd prime order in $G$. Then one of the following holds.

(i) There exists $g \in G$ such that $\left\langle x, x^{g}\right\rangle$ is not solvable.

(ii) $p=3$ and $\left(x, G_{0}\right)$ belongs to a short list of exceptions given in Table 1, Moreover, there exist $g_{1}, g_{2} \in G$ such that $\left\langle x, x^{g_{1}}, x^{g_{2}}\right\rangle$ is not solvable, unless $G_{0} \cong P S U(n, 2), P S p(2 n, 3)$. In any case, there exist $g_{1}, g_{2}, g_{3} \in G$ such that $\left\langle x, x^{g_{1}}, x^{g_{2}}, x^{g_{3}}\right\rangle$ is not solvable.

Corollary 2. Let $G$ be an almost simple group, and suppose that $x \in G$ has prime order $p \geq 5$. Suppose that $x$ is contained in the solvable radical of all proper subgroups $M$ containing $x$. Then there exists $g \in G$ such that $\left\langle x, x^{g}\right\rangle=G$.

Proof. By Theorem A*, there exists $g \in G$ such that $\left\langle x, x^{g}\right\rangle$ is not solvable. If $\left\langle x, x^{g}\right\rangle \neq G$, then it is contained in some maximal subgroup $M$. However, the hypothesis implies that $x \in O_{\infty}(M)$, which would mean that $\left\langle x, x^{g}\right\rangle$ would be solvable. Thus $\left\langle x, x^{g}\right\rangle=G$.

Clearly, we only need to check that the hypothesis in the corollary is true for all maximal subgroups. Indeed, if $x \in M$ and $M<M^{\prime}<G$, then $\left\langle x^{M^{\prime}}\right\rangle$ is solvable; therefore $\left\langle x^{M}\right\rangle$ is solvable and thus $x \in O_{\infty}(M)$.

\section{Preliminaries}

Let $\bar{G}$ be a simple classical algebraic group of adjoint type over the algebraic closure of $\mathbb{F}_{q}$. Let $\sigma$ be a Frobenius morphism of $\bar{G}$ such that $\bar{G}_{\sigma}:=\left\{g \in \bar{G}: g^{\sigma}=g\right\}$ is a finite almost simple classical group over $\mathbb{F}_{q}$. Write $G_{0}$ for the socle of $\bar{G}_{\sigma}$ and note that $\bar{G}_{\sigma}$ is the group $\operatorname{Inn} \operatorname{diag}\left(G_{0}\right)$ of inner diagonal automorphisms of $G_{0}$. A collection of lemmas, definitions, and theorems is listed below, which will be very useful in the sequel. 
Lemma 3. Let $x \in \bar{G}_{\sigma}$ have odd prime order $r$. Define $(G, \hat{G})$ as follows:

\begin{tabular}{|c|ccc|}
\hline$G_{0}$ & $P S L_{n}^{\epsilon}(q)$ & $P S p_{n}(q)$ & $P \Omega_{n}^{\epsilon}(q)$ \\
\hline$(G, \hat{G})$ & $\left(\bar{G}_{\sigma}, G L_{n}^{\epsilon}(q)\right)$ & $\left(G_{0}, S p_{n}(q)\right)$ & $\left(G_{0}, \Omega_{n}^{\epsilon}(q)\right)$ \\
\hline
\end{tabular}

(a) Then one of the following holds: (i) $x$ lifts to an element $\hat{x} \in \hat{G}$ of order $r$ such that $\left|x^{G}\right|=\left|\hat{x}^{\hat{G}}\right|$.

(ii) $G_{0}=P S L_{n}^{\epsilon}(q), r \mid \operatorname{gcd}(q-\epsilon, n)$ and $x$ is $\bar{G}$-conjugate to $\left[I_{\frac{n}{r}}, \omega I_{\frac{n}{r}}, \ldots\right.$, $\left.\omega^{r-1} I_{\frac{n}{r}}\right]$, where $\omega$ is a primitive $r$ th root of unity.

(b) If $r \nmid q$, then $x^{G_{0}}=x^{\bar{G}_{\sigma}}$.

Proof. See [Bur04, 3.11] and [GLS98, 4.2.2(j)].

Definition 1. Let $\mathcal{A}$ be the set of pairs $(x, H)$ such that:

(i) $x$ is an element of odd prime order contained in a group $H$;

(ii) $H / O_{\infty}(H)$ is almost simple;

(iii) $x$ is not contained in $O_{\infty}(H)$;

(iv) $\left(x, H / O_{\infty}(H)\right)$ is not one of the examples in Table 1

Lemma 4. (a) If $x \in G$ is an inner-diagonal automorphism of $G_{0}$ and $\left|x^{G_{0}}\right|=$ $\left|x^{\bar{G}_{\sigma}}\right|$, then it suffices to take $G=\bar{G}_{\sigma}$.

(b) If $y$ is some Aut $\left(G_{0}\right)$-conjugate of $x$ and there exists $l \in G_{0}$ such that $\left\langle y, y^{l}\right\rangle$ is not solvable, then there exists $l^{\prime} \in G_{0}$ such that $\left\langle x, x^{l^{\prime}}\right\rangle$ is not solvable.

(c) If $x$ is contained in $H$, a proper subgroup of $G$, and $(x, H) \in \mathcal{A}$, then $G$ cannot be a minimal counterexample to Theorem $A^{*}$.

Proof. (a) Suppose that the theorem is true for $\bar{G}_{\sigma}$. If $x$ is contained in $G$, then $x \in \bar{G}_{\sigma}$ and so there exists $g \in \bar{G}_{\sigma}$ such that $\left\langle x^{g}, x\right\rangle$ is not solvable. But then there exists $g_{1} \in G_{0}$ such that $x^{g_{1}}=x^{g}$ by the condition.

(b) Suppose that $y=x^{g}$ for some $g \in \operatorname{Aut}\left(G_{0}\right)$. Then $\left\langle y, y^{l}\right\rangle^{g^{-1}}=\left\langle x, y^{l g^{-1}}\right\rangle=$ $\left\langle x, x^{l^{\prime}}\right\rangle$ since $l g^{-1}=g^{-1} g l g^{-1}=g^{-1} l^{\prime}$.

(c) Trivial.

Lemma 5. Let $X_{1}, \ldots, X_{k}$ be representatives for the conjugacy classes of maximal subgroups containing $x$. Let $n_{i}$ be the number of conjugates of $X_{i}$ that contain $x$. If

$$
\left|x^{G}\right|^{2}>\sum_{i} n_{i}\left|x^{G} \cap X_{i}\right|=\sum\left|x^{G} \cap X_{i}\right|^{2}\left[G: X_{i}\right],
$$

then there exists $g \in G$ such that $\left\langle x, x^{g}\right\rangle=G$.

Proof. Let $X_{i 1}, \ldots, X_{i n_{i}}$ be the conjugates of $X_{i}$ that contain $x$. The aim is to show that $x^{G}$ cannot be contained in $\bigcup_{i, j} X_{i j}$, since this proves the lemma. It is not hard to show that $n_{i} /\left[G: X_{i}\right]=\left|x^{G} \cap X_{i}\right| /\left|x^{G}\right|$. It then follows that

$$
\begin{aligned}
\left|x^{G} \cap \cup_{i, j} X_{i j}\right| & \leq \sum_{i} n_{i}\left|x^{G} \cap X_{i}\right| \\
& =\sum\left|x^{G} \cap X_{i}\right|^{2}\left[G: X_{i}\right] /\left|x^{G}\right|
\end{aligned}
$$

and so if $x^{G}$ were contained in $\bigcup_{i, j} X_{i j}$, then

$$
\begin{aligned}
\left|x^{G}\right|=\left|x^{G} \cap \bigcup_{i, j} X_{i j}\right| & \leq \sum_{i} n_{i}\left|x^{G} \cap X_{i}\right| \\
& =\sum\left|x^{G} \cap X_{i}\right|^{2}\left[G: X_{i}\right] /\left|x^{G}\right| .
\end{aligned}
$$


However, this implies that

$$
\left|x^{G}\right|^{2} \leq \sum_{i} n_{i}\left|x^{G} \cap X_{i}\right|=\sum\left|x^{G} \cap X_{i}\right|^{2}\left[G: X_{i}\right],
$$

which contradicts the hypothesis.

Remark. If

$$
|G| /\left|C_{G}(x)\right|^{2}>\sum_{i}\left|x^{G} \cap X_{i}\right|,
$$

then the conclusion of the theorem holds.

Lemma 6. Let $G_{0}$ be a simple group of Lie type and suppose that $G$ satisfies $G_{0} \unlhd G \leq \operatorname{Inndiag}\left(G_{0}\right)$.

(i) Suppose that $x \in G$ is unipotent and $P_{1}$ and $P_{2}$ are distinct maximal parabolic subgroups containing a common Borel subgroup, with unipotent radicals $U_{1}$ and $U_{2}$, respectively. Then there exists $i \in\{1,2\}$ such that $x$ is $G$-conjugate to an element of $P_{i} \backslash U_{i}$.

(ii) Suppose that $x \in G$ is semisimple and is contained in a parabolic subgroup of $G$. Suppose further that the Lie rank of $G_{0}$ is at least 2. Then there exists a maximal parabolic subgroup $P$ with a Levi complement $J$ such that $x$ is conjugate to an element of $J$ not centralized by any component of $J$.

Proof. See GS03, Lemma 2.2].

Theorem 4. Let $G$ be an almost simple group and let $x \in G$ with $x \neq 1$. If $x^{G} \subseteq M_{1} \cup M_{2}$ for subgroups $M_{1}$ and $M_{2}$ of $G$, then $G_{0}$ is contained in $M_{i}$ for $i=1$ or 2 .

Proof. See Gur98, Theorem 2.1].

To begin the proof of Theorem $\mathrm{A}^{*}$, let $(x, G)$ be a minimal counterexample. Then $G$ is almost simple with socle $G_{0}$. If $p \geq 5$, then Theorem A holds for any group containing fewer elements than $G$.

\section{Alternating groups}

Suppose that $G_{0}=A_{n}$. Then $x$ is contained in $A_{n}$ since it has odd order. Firstly, consider the case where $p \geq 5$. The cycle structure of $x$ will consist only of $p$-cycles. So it suffices to assume that $x=(12 \ldots p) \sigma$ for some $\sigma \in \operatorname{Alt}\{p+1, \ldots, n\}$. Observe that if $g:=(123)$, then $x g x^{-1} g^{-1}=(2 p 3)$. Thus $\left\langle x, x^{g}\right\rangle$ contains Alt $\{1,2, \ldots, p\}$ since a primitive permutation group of degree $p \geq 5$ containing a 3 -cycle contains $A_{p}$ (see [Wie64, Theorem 13.9] for example). So $(x, G)$ cannot be a counterexample in this case.

Now suppose that $p=3$. Then the cycle structure of $x$ consists of only 3 cycles. If $x$ is the product of more than one 3-cycle, then it suffices to assume that $G=A_{6}$ and $x$ is the product of two 3-cycles. But then $x$ is conjugate to a 3-cycle in $\operatorname{Aut}\left(A_{6}\right)$. Thus we may assume that $x$ is a 3 -cycle in $A_{5}$ and without loss of generality, that $x=(123)$. If $g:=(14253)$, then $x^{g}=(451)$. Thus, $x x^{g}=(12345)$ and $\left\langle x, x^{g}\right\rangle \cong A_{5}$.

$$
\text { 5. } P S L(n, q)
$$

If $G_{0} \cong P S L(n, q)$, then it is convenient to treat the cases where $n=2$ and $n \geq 3$ separately. 
5.1. $G_{0} \cong P S L(2, q)$. Suppose that $x$ is in $\operatorname{Inndiag}(P S L(2, q)) \cong P G L(2, q)$. Since $x$ has odd order, it must lie in $P S L(2, q)$.

5.1.1. $x \in \operatorname{Inndiag}(P S L(2, q))$ and $p \mid q$. If $p \mid q$ and $p \geq 5$, then it suffices to assume that $x$ is contained in $P S L(2, p)$. Consider the possibilities for the maximal subgroups of $P S L(2, p)$ containing $\left\langle x, x^{g}\right\rangle$, which are described in GLS98, Theorem 6.5.1]. By the order of $x$ and since $(x, G)$ is a minimal counterexample, the only type of maximal subgroup possible is a Borel subgroup, $B$. Now since $p \mid q, x$ and $x^{g}$ must lie inside the kernel $K$ of $B$ which is (elementary) abelian. So any $p$-elements lying in a common Borel subgroup must commute. Thus, since there must exist a conjugate of $x$ that does not commute with $x$ (otherwise $\left[x^{G}, x^{G}\right]=1$ and $G_{0}$ would be abelian) there exists $g \in G$ such that $\left\langle x, x^{g}\right\rangle=P S L(2, p)$, which is not solvable for $p \geq 5$.

If $p=3$, then $q=3^{a}$, where $a>1$ and since $x \in P S L(2, q)$, it suffices to assume that $G=P S L(2, q)$. Now $A_{6} \cong P S L(2,9)$, so let us assume that $q>9$. If $q=3^{a}$ and $a$ is not prime, then there exists a conjugate $x^{g}$ of $x$ that is contained in a subfield subgroup $H$ with $\left(x^{g}, H\right)$ in $\mathcal{A}$. So $a$ must be an odd prime. Now we may assume that

$$
x=\left(\begin{array}{ll}
1 & 1 \\
0 & 1
\end{array}\right) .
$$

There are two classes of transvections in $G$, and since -1 is not a square in $\mathbb{F}_{q}, x$ and $x^{-1}$ are not conjugate. Thus if we let

$$
y:=\left(\begin{array}{ll}
1 & 0 \\
s & 1
\end{array}\right),
$$

then $x$ or $x^{-1}$ is conjugate to $y$. So there exists $g \in G$ such that $\left\langle x, x^{g}\right\rangle$ contains

$$
x y=\left(\begin{array}{cc}
1+s & 1 \\
s & 1
\end{array}\right)
$$

which is semisimple and has trace $s+2$. In particular we can choose $s$ so that $x y$ has order $\frac{q+1}{2}$ and an inspection of the maximal subgroups of $G$ shows that $\left\langle x, x^{g}\right\rangle=G$.

5.1.2. $x \in \operatorname{Inndiag}(P S L(2, q))$ and $p \nmid q$. Suppose now that $p \nmid q$. Then either $p \mid q-1$ or $p \mid q+1$. If $p \mid q-1$, then $x$ is contained in a split torus. Examining the character table of $\operatorname{PSL}(2, q)$ shows that for an element $z$ of order $(q+1) /(2, q-1)$,

$$
\sum_{\chi \in \operatorname{Irr}\left(G_{0}\right)} \frac{\overline{\chi(z)}|\chi(x)|^{2}}{\chi(1)} \neq 0,
$$

so there exists $g \in G$ such that $[x, g]$ has order $(q+1) /(2, q-1)$. That is, $[x, g]$ generates a non-split torus. It follows that $\left\langle x, x^{g}\right\rangle$ generates $P S L(2, q)$. Indeed, $\left\langle x, x^{g}\right\rangle$ contains an irreducible torus, and it also contains $x$, which does not normalize this torus. An inspection of the maximal subgroups of $P S L(2, q)$ yields that $\left\langle x, x^{g}\right\rangle$ must generate the whole group for $q \geq 11$, and $q=8$. It suffices to assume that $q \neq 4,5$ or 9 since in those cases $G$ is isomorphic to an alternating group. When $q=7$, the normalizer of a non-split torus is not maximal, but is contained in subgroups isomorphic to $S_{4}$. However, since $p \mid q-1,\left\langle x, x^{g}\right\rangle$ cannot be contained in $S_{4}$ since $S_{4}$ does not contain two elements of order 3 whose product has order 4 . 
If $p \mid q+1$, then the character table implies that there exists $g \in G$ such that $[x, g]$ has order $(q-1) /(2, q-1)$. Thus $\left\langle x, x^{g}\right\rangle$ contains a split torus, and since $p \mid q+1$, it acts irreducibly. Therefore, an inspection of the maximal subgroups shows that for $q \geq 13$ and $q=8,\left\langle x, x^{g}\right\rangle=P S L(2, q)$. Again, the cases when $q=4$, 5 , and 9 do not concern us. Also note that $q \neq 7$ since $p \mid q+1$. If $q=11$, then $\left\langle x, x^{g}\right\rangle$ contains a maximal split torus and acts irreducibly. The list of maximal subgroups then implies that either $\left\langle x, x^{g}\right\rangle=P S L(2, q)$ or $A_{5}$. There are no other possibilities for $x \in \operatorname{Inndiag}(P S L(2, q))$.

5.1.3. $x$ an outer automorphism of $\operatorname{PSL}(2, q)$. Suppose that $x$ is not contained in Inndiag $\left(G_{0}\right)$. Then by GL83, 7.2], and since $x$ has odd order, there exists an element $g \in P G L(2, q)$ such that $x^{g}$ is a standard field automorphism. So it suffices to assume that $x$ is a standard field automorphism by Lemma 4 , and moreover, that $G=\left\langle G_{0}, x\right\rangle$. Write $q=q_{1}^{p}$ and consider the set $\Gamma=\left\{y \in x^{G_{0}} \mid\langle x, y\rangle \neq G\right\}$. The aim is to bound the cardinality of $\Gamma$ and show that this is smaller than $\left|x^{G_{0}}\right|$. Now if $y \in \Gamma$, then consider the possibilities for subgroups $H$ of $G_{0}$ containing $\langle x, y\rangle \cap G_{0}$. Observe that $\langle x, y\rangle \cap G_{0}$ cannot be dihedral. Indeed, since a dihedral group has a characteristic cyclic subgroup of index $2, K$ say, $K$ would be normal in $\langle x, y\rangle$. Now $\langle x, y\rangle / K$ has a normal subgroup of order 2 and a subgroup of order $p$, which is normal since it has index 2. So, $\langle x, y\rangle / K$ is abelian of order $2 p$, but this is impossible since it is generated by two elements of order $p$. Thus, it suffices to assume that $H$ is a Borel subgroup, a cyclic group of order $(q+1) /(2, q-1)$, or a subfield subgroup. Since $p$ is odd, any $A_{5}$ or $S_{4}$ will be contained in a subfield subgroup and any cyclic group of order $(q-1) /(2, q-1)$ will be contained in a Borel subgroup. Now let $H$ be a Borel, non-split torus or subfield subgroup of the form $L\left(2, q^{1 / r}\right)$, where $r$ is a prime distinct from $p$. Observe that we may assume that there are no subfield subgroups of the form $L\left(2, q^{1 / r}\right)(r \neq p)$ since some conjugate of $x$ will be a non-trivial field automorphism of the simple subgroup $L\left(2, q^{1 / r}\right)$ contradicting the minimality of $(x, G)$. Now the conjugates of $H$ fixed by $x$ form one $C_{G_{0}}(x)$ orbit. This follows from the fact that any two conjugates of $x$ in $H\langle x\rangle$ are in fact conjugate by an element of $H$, which is a consequence of Lang's Theorem (see [GL83, 7.2]). So if $k$ is the number of conjugates of $H$ fixed by $x$, then

$$
\begin{aligned}
\mid\left\{y \in x^{G_{0}}:\langle x, y\rangle \cap G_{0} \text { is contained in a conjugate of } H\right\} \mid & \leq\left|x^{H}\right| . k \\
& =\frac{|H|\left|C_{G_{0}}(x)\right|}{\left|C_{H}(x)\right|^{2}} .
\end{aligned}
$$

Moreover $x$ does not fix any non-trivial conjugate of $C_{G_{0}}(x)=P S L\left(2, q^{1 / p}\right)$, so

$$
\mid\left\{y \in x^{G_{0}}:\langle x, y\rangle \cap G_{0} \text { is contained in some conjugate of } C_{G_{0}}(x)\right\}|\leq| C_{G_{0}}(x) \mid .
$$

Therefore if the representatives for the conjugacy classes of the subgroups above are denoted by $H_{1}, \ldots, H_{m}, H_{m+1}:=C_{G_{0}}(x)$, then

$$
\begin{aligned}
|\Gamma| & =\mid\left\{y \in x^{G_{0}}: G_{0} \cap\langle x, y\rangle \text { is contained in some conjugate of some } H_{i}\right\} \mid \\
& \leq\left|C_{G_{0}}(x)\right|+\sum_{i=1}^{m}\left|H_{i}\right|\left|C_{G_{0}}(x)\right| /\left|C_{H_{i}}(x)\right|^{2} .
\end{aligned}
$$

If $q_{0}:=q^{1 / p}$, then

$$
\begin{aligned}
\left|H \| C_{G_{0}}(x)\right| /\left|C_{H}(x)\right|^{2} & =\left(q_{0}^{p}+1\right) q_{0}\left(q_{0}^{2}-1\right) /\left(q_{0}+1\right)^{2} \\
& =\left(q_{0}^{p}+1\right) q_{0}\left(q_{0}-1\right) /\left(q_{0}+1\right)
\end{aligned}
$$


when $H$ is a non-split torus. Similarly if $H$ is a Borel subgroup, then

$$
|H|\left|C_{G_{0}}(x)\right| /\left|C_{H}(x)\right|^{2}=q_{0}^{p}\left(q_{0}^{p}-1\right)\left(q_{0}+1\right) / q_{0}\left(q_{0}-1\right) .
$$

So,

$$
|\Gamma| \leq q_{0}\left(q_{0}^{2}-1\right)+\frac{q_{0}^{p}\left(q_{0}^{p}-1\right)\left(q_{0}+1\right)}{q_{0}\left(q_{0}-1\right)}+\frac{\left(q_{0}^{p}+1\right) q_{0}\left(q_{0}-1\right)}{\left(q_{0}+1\right)} .
$$

However, $\left|x^{G_{0}}\right|=\left|G_{0}\right| /\left|C_{G_{0}}(x)\right|=\frac{q_{0}^{p}\left(q_{0}^{2 p}-1\right)}{q_{0}\left(q_{0}^{2}-1\right)}$ and $q \geq 8$, so it follows that $\left|x^{G_{0}}\right|>$ $|\Gamma|$, as required. Thus, if $x$ is an outer automorphism of $P S L(2, q)$, then $(x, G)$ cannot be a minimal counterexample.

\section{Outer Automorphisms}

If $(x, G)$ is a minimal counterexample and $x$ is an outer automorphism of $G_{0}$, then the work for $G_{0}=\operatorname{PSL}(2, q)$ allows a considerable narrowing of the possibilities for $G_{0}$. This is demonstrated in Lemma 7 below.

Lemma 7. If $x$ is an outer automorphism of $G_{0}$ that is not inner-diagonal and $(x, G)$ is a minimal counterexample, then $G_{0}$ is a Suzuki-Ree group.

Proof. Since $x \notin \operatorname{Inndiag}\left(G_{0}\right)$ and $x$ has odd prime order, either $x$ is a field automorphism or, $G_{0} \cong D_{4}(q)$ or ${ }^{3} D_{4}(q)$ and $x$ is a graph or graph-field automorphism. Since the case where $G_{0} \cong P S L(2, q)$ has already been eliminated, the Lie rank is at least 2. If $x$ is a field automorphism, then by GL83, 7.2] and Lemma 4 it suffices to assume that $x$ is a standard field automorphism. So if $G_{0}$ is not a Suzuki-Ree group, then $x$ will act non-trivially as a field automorphism on a fundamental $S L_{2}$-subgroup, by [GLS98, Theorem 3.2.8]. So $(x, G)$ cannot be a minimal counterexample.

If $G_{0} \cong{ }^{3} D_{4}(q)$ and $x$ is a graph automorphism of order 3, then GL83, 9.1] describes the conjugacy classes of such elements. Let $\gamma$ be the standard triality automorphism and $g=\overline{h_{\beta_{0}}(\omega)}$, where $\omega$ is a primitive cube root of unity and $\beta_{0}$ is the $\gamma$-invariant fundamental root. Thus, if $3 \nmid q$, then it suffices to assume that $x$ is either $\gamma$ or $g \gamma$. Also, if $3 \mid q$, then it suffices to assume that $x$ is either $\gamma$ or $x_{\beta}(1) \gamma$, where $\beta$ is the highest root. In all cases, $x$ normalizes the maximal parabolic corresponding to $\beta_{0}$. Moreover $x$ acts non-trivially on the Levi complement in all these cases and so $(x, G)$ cannot be a minimal counterexample. The only case left is where $G_{0} \cong D_{4}(q)$ and $x$ is a graph or field-graph automorphism of order 3, in which case, using GL83, it suffices to assume that $x$ is either the standard triality (and $C_{G_{0}}(x)=G_{2}(q)$ ) or it normalizes but does not centralize a subgroup isomorphic to $G_{2}(q)$. In the latter case $x$ induces a non-trivial automorphism on $G_{2}(q)$, so $(x, G)$ cannot be a minimal counterexample. In the former case, since $G_{2}(q)$ does not contain a Sylow 3-subgroup, $x$ normalizes more than one conjugate of $G_{2}(q)$. Since it only centralizes one $G_{2}(q)$ subgroup, it follows that $x$ induces a non-trivial automorphism on some subgroup isomorphic to $G_{2}(q)$, and so $(x, G)$ cannot be a minimal counterexample.

\section{7. $P S L(n, q), n \geq 3$}

7.1. $x \in P G L(n, q), p \nmid q, n \geq 3$. Now suppose that $(x, G)$ is a minimal counterexample with $G_{0}=P S L(n, q)$ and $n \geq 3$. 
Lemma 8. For $n \geq 3$, if one can lift $x$ to an element of order $p$ in $G L(n, q)$ and $x$ does not act irreducibly, then $(x, G)$ cannot be a minimal counterexample.

Proof. Suppose that one can lift $x$ to an element of $G L(n, q)$ of order $p$. Now the minimal polynomial $m_{x}(t)$ of $x$ divides $\left(t^{p}-1\right)$, so suppose that $\left(t^{p}-1\right) /(t-1)$ factors into irreducibles $g_{1}(t) \ldots g_{k}(t)$. Then each non-linear $g_{i}(t)$ is the minimal polynomial of some primitive $p$ th root of unity $\zeta_{p}$. Thus

$$
\operatorname{deg} g_{i}(t)=\left[\mathbb{F}_{q}\left(\zeta_{p}\right): \mathbb{F}_{q}\right] .
$$

But $\mathbb{F}_{q}\left(\zeta_{p}\right)$ is just the finite field of $q^{e}$ elements, where $e$ is the smallest positive integer such that $p \mid q^{e}-1$. So all of the $g_{i}(t)$ 's have degree $e$. Now $m_{x}(t)$ is a product of some $g_{i}(t)$ 's and possibly $t-1$. By considering the rational canonical form of $x$, it is clear that there is an $e$-dimensional subspace $U$ of $V$ on which $x$ acts invariantly, non-trivially and irreducibly. If $2 \leq e<n$, then consider the induced transformation of $U, x_{U}$ so that $x_{U} \in G L(e, q)$. Now observe that if $(e, q) \neq(2,2)$ or $(2,3)$, then $\left(x_{U}, G L(e, q)\right) \in \mathcal{A}$. If $(e, q)=(2,3)$, then $p$ would be 2. So the only case of concern is $(e, q)=(2,2)$ and then for $n \geq 4$ one can just reduce to the case where $G_{0}=P S L(4,2)$. However $P S L(4,2) \cong A_{8}$ and $P S L(3,2) \cong P S L(2,7)$, which have already been eliminated. If $e=1$, then since $p \mid q-1, q \geq 4$. Now $x$ will act non-trivially on a 2 -dimensional subspace $U^{\prime}$; thus $x_{U^{\prime}} \in G L(2, q)$ and $\left(x_{U^{\prime}}, G L(2, q)\right) \in \mathcal{A}$. So unless $e=n \geq 3,(x, G)$ cannot be a minimal counterexample.

Now observe that the proof above shows that if $(x, P G L(n, q))$ is a minimal counterexample and $x$ lifts to an element of order $p$ in $G L(n, q)$, then $p$ is a primitive prime divisor of $q^{n}-1$ and $x$ acts irreducibly. Also, if $x$ acts irreducibly and $n$ is not prime, then some conjugate of $x$ is contained in a field extension subgroup $P G L\left(\frac{n}{r}, q^{r}\right)$. Thus, if $(x, P G L(n, q))$ is a minimal counterexample, then $n$ is prime.

The results in GPPS99 state that any subgroup of $G L(n, q)$ that has order divisible by a primitive prime divisor of $q^{e}-1$ must be one of nine types (2.1-2.9). The results of GPPS99. will be used frequently and are summarized in Table 2 . The notation of GPPS99 will be used, namely, that the element of $G L(d, q)$ that is a primitive prime divisor of $q^{e}-1$ will be referred to as a $\operatorname{ppd}(d, q, e)$-element. The only elements that are of interest are $\operatorname{ppd}(n, q, n)$-elements, where $n$ is (an odd) prime. So what are the possibilities for a maximal subgroup $M$ of $G L(n, q)$ containing $x$ ?

Lemma 9. Suppose that $x$ is a ppd(n,q,n)-element contained in a subgroup $M$ of $G$, where $G$ is a classical group of dimension $n \geq 3$ and $(x, G)$ is a minimal counterexample. Then $p \geq 5$ and $M$ cannot be of type 2.2, 2.3, 2.4(a), 2.6, 2.7, 2.8, or 2.9 .

Proof. Firstly, if $p=3$, then since $p \nmid q$, Fermat's Little Theorem implies that $p \mid q^{2}-1$; thus $p$ cannot be a primitive prime divisor of $q^{n}-1$ for $n \geq 3$. If $G$ is a classical group, then $M \leq G \leq G L(n, q)$ for some $q$, and so $M$ must be one of the examples in GPPS99. All of the subgroups $M$ of type 2.6-2.9 are almost simple modulo scalars, so it suffices to check that $(x, M /(M \cap Z)) \in \mathcal{A}$. 
TABLE 2. Summary of descriptions in [GPPS99] of subgroup types containing $\operatorname{ppd}(d, q, e)$-elements

\begin{tabular}{|c|c|c|}
\hline Type & Rough description & Conditions on $d, q, e$ \\
\hline Classical (2.1(a)) & $S L\left(d, q_{0}\right) \unlhd M$ & $p$ a $\operatorname{ppd}\left(q_{0}, d, e\right)$-element \\
\hline Classical (2.1(b)) & $S p\left(d, q_{0}\right) \unlhd M$ & $\begin{array}{l}d, e \text { both even; } \\
p \text { a } \operatorname{ppd}\left(q_{0}, d, e\right) \text {-element }\end{array}$ \\
\hline Classical $(2.1(\mathrm{c}))$ & $S U\left(d, q_{0}\right) \unlhd M$ & $q_{0}$ a square; $e$ odd; \\
\hline Classical (2.1(d)) & $\Omega^{\epsilon}\left(d, q_{0}\right) \unlhd M$ & $\begin{array}{l}\epsilon= \pm \text { when } d \text { even; } \\
\epsilon=0 \text { when } d q \text { is odd; } \\
e \text { even; } \\
p \text { a } \operatorname{ppd}\left(q_{0}, d, e\right) \text {-element }\end{array}$ \\
\hline Reducible (2.2) & $M$ reducible & \\
\hline Imprimitive (2.3) & $M \leq G L(1, q)$ wr $S_{d}$ & $p=e+1 \leq d$ \\
\hline Extension Field $(2.4(\mathrm{a}))$ & $M \leq G L\left(1, q^{d}\right) \cdot d$ & $p=d=e+1$ \\
\hline Extension Field (2.4(b)) & $M \leq G L\left(d / b, q^{b}\right) \cdot b$ & $b \mid \operatorname{gcd}(d, e)$ \\
\hline Symplectic type (2.5) & & $\begin{array}{l}d=2^{a} \\
q \text { odd not a square; } \\
p=d+1=e+1 \text { or } \\
p=d-1=e+1\end{array}$ \\
\hline Nearly simple $(2.6-2.9)$ & $M /(M \cap Z)$ simple & $\begin{array}{l}\text { Possibilities listed } \\
\text { in tables in GPPS99 }\end{array}$ \\
\hline
\end{tabular}

If $M$ is of type 2.6 or 2.7 , then $F^{*}(M /(M \cap Z)) \cong A_{d}$ for some $d$, or a sporadic group and so $(x, M /(M \cap Z)) \in \mathcal{A}$. The only $\operatorname{ppd}(n, q, n)$-elements in the type 2.8 examples, i.e., $(M /(M \cap Z)) \in \operatorname{Lie}\left(q_{0}\right)$, are with $M^{(\infty)}=G_{2}\left(q_{1}\right), q_{0}=2$ and $M^{(\infty)}={ }^{2} B_{2}\left(q_{1}\right), q_{0}=2$, but these occurrences must all lie in $\mathcal{A}$. Similarly, all of the type 2.9 subgroups in GPPS99, Tables 7 and 8] coincide with elements of $\mathcal{A}$. Since $x$ acts irreducibly it cannot be contained in a reducible subgroup of type 2.2 and it cannot be contained in a type 2.3 example since these are only examples for $\operatorname{ppd}(d, q, e)$-elements, where $e+1 \leq d$. Similarly $x$ cannot be contained in a 2.4(a) type subgroup since these are only examples for $\operatorname{ppd}(d, q, e)$-elements, where $e+1=d$.

Suppose that $x$ is contained in a classical example of type 2.1. By KL90 and GPPS99, since $n \geq 3$, all of the classical examples containing $\operatorname{ppd}(n, q, n)$-elements are almost simple modulo scalars. So if $x$ is contained in a type 2.1 subgroup $M$, then $(x, G)$ cannot be a minimal counterexample since $p \geq 5$. The symplectic type examples (2.5) only occur as subgroups of $G L\left(2^{a}, q\right)$, but it is assumed that $n$ is an odd prime. Therefore, the only possibilities for subgroups $M$ containing $x$ are the extension field examples of type (2.4(b)). Since $n$ is prime, $M$ must be of type $G L\left(1, q^{n}\right) . n$. Moreover, if $p \mid n$, then $p=n$ since $n$ is prime. However, $p \nmid q^{p}-1$ so $p \nmid n$. Thus, $x$ must lie inside the Singer cycle $G L\left(1, q^{n}\right)$. Furthermore, $C_{G L(n, q)}(x)=G L\left(1, q^{n}\right)$; thus $x$ can only lie in one such maximal subgroup and applying Theorem 4 yields that $(x, G)$ cannot be a minimal counterexample.

Lemma 10. If $x$ does not lift to an element of order $p$ in $G L(n, q)$, then $(x, G)$ cannot be a minimal counterexample. 
Proof. Suppose that $x$ does not lift to an element of order $p$ in $G L(n, q)$. Now $x^{p}$ is central, so $x$ satisfies the polynomial $p(t):=t^{p}-\lambda$. Now $p(t)$ is irreducible over $\mathbb{F}_{q}$. For $p \mid(q-1)$, since $x$ does not lift, thus any field containing a root $\alpha$ of $p(t)$ would be a splitting field for $p(t)$. So the degree of any irreducible factor of $p(t)$ is the degree of the splitting field extension over $\mathbb{F}_{q}$. However, $p(t)$ has prime degree and so it is either irreducible or it splits completely. It cannot split completely; otherwise $\lambda$ would have $p$ th roots and $x$ would lift to an element of order $p$. Thus, the irreducible module for $\langle x\rangle$ has dimension $p$ and so it suffices to deal with the case where $n=p$. So let $v$ be a vector in $V$ and consider the action of $x$ on $v$. The vectors $v, x v, x^{2} v, \ldots,\left(x^{p-1}\right) v$ form a basis for $V$ since $x$ acts irreducibly. Moreover $x^{p} v=\lambda v$. So $x$ is contained in a subgroup of type $G L(1, q) \imath S_{p}$ and $x$ acts as a $p$-cycle in the $S_{p}$. So for $p \geq 5$, we have shown that $(x, G)$ cannot be a minimal counterexample. Now suppose that $p=3$. Then it suffices to assume that $x$ has the form

$$
\left(\begin{array}{lll}
0 & 0 & \lambda \\
1 & 0 & 0 \\
0 & 1 & 0
\end{array}\right)
$$

Now let $t^{2}-\mu_{2} t-\mu_{1}$ be an irreducible polynomial in $\mathbb{F}_{q}[t]$, such that $\left(\begin{array}{ll}0 & \mu_{1} \\ 1 & \mu_{2}\end{array}\right)$ has order $q^{2}-1$. Now $x$ is conjugate to

$$
y:=\left(\begin{array}{ccc}
0 & 0 & -\mu_{1}^{-1} \lambda \\
0 & \mu_{1} & \mu_{2}^{-1}\left(\mu_{1}^{-1} \lambda-\mu_{1}^{2}\right) \\
1 & \mu_{2} & -\mu_{1}
\end{array}\right),
$$

and therefore

$$
x^{-1} y:=\left(\begin{array}{ccc}
0 & \mu_{1} & \mu_{2}^{-1}\left(\mu_{1}^{-1} \lambda-\mu_{1}^{2}\right) \\
1 & \mu_{2} & -\mu_{1} \\
0 & 0 & -\mu_{1}^{-1}
\end{array}\right)
$$

has order a multiple of $q^{2}-1$. Thus, by GLS98, Theorem 6.5.3], $\langle x, y\rangle$ is not solvable and hence $(x, G)$ cannot be a minimal counterexample. The case where $p \mid q$ is considered in the next section.

\section{UNIPOTENT ELEMENTS}

Lemma 11. Suppose that $G_{0}$ is a simple group of Lie type and suppose that $x \in G_{0}$ is unipotent of order $p$. If $G_{0}$ is defined over $\mathbb{F}_{q}$ and $q \neq 3$, then $(x, G)$ cannot be a minimal counterexample unless $G_{0}=\operatorname{PSU}(3, q)$ or ${ }^{2} G_{2}(q)$.

Proof. The case where $G_{0}=P S L(2, q)$ has already been done. Since $p$ is an odd prime, $G_{0} \neq{ }^{2} B_{2}(q)$ or ${ }^{2} F_{4}(q)$. In the remaining cases, by Lemma 6, for any two maximal parabolic subgroups $P_{1}$ and $P_{2}$ (containing a common Borel subgroup) there exists a conjugate of $x$ that is contained in $P_{i} \backslash U_{i}$ for either $i=1$ or 2 . The parabolic subgroups can be chosen so that the Levi complement has only one component, and since $q \geq 5$, it will always be almost simple. It follows that since $\left(x U_{i}, P_{i} / U_{i}\right)$ will be contained in $\mathcal{A},(x, G)$ cannot be a minimal counterexample. Table 3 describes the parabolic subgroups to choose and the possibilities for the Levi complement. 
TABLE 3. Maximal parabolic subgroups and their Levi complements used in Lemma 11

\begin{tabular}{|c|c|c|}
\hline$G_{0}$ & $\begin{array}{c}\text { Nodes corresponding to } P_{1} \text { and } P_{2} \\
\text { (Bourbaki notation used } \\
\text { where node is specified) }\end{array}$ & Levi complement type \\
\hline$A_{l}(q), l \geq 2$ & end nodes & $A_{l-1}(q)$ \\
$B_{2}(q)$ & end nodes & $A_{1}(q)$ \\
$B_{l}(q), l \geq 3$ & end nodes & $B_{l-1}(q), A_{l-1}(q)$ \\
$C_{l}(q), l \geq 3$ & end nodes & $C_{l-1}(q), A_{l-1}(q)$ \\
$D_{4}(q)$ & any end nodes & $A_{3}(q)$ \\
$D_{l}(q), l \geq 5$ & any end nodes & $D_{l-1}(q), A_{l-1}(q)$ \\
${ }^{2} A_{l}(q), l \geq 3, l$ odd & end and middle node & $A_{(l-1) / 2}\left(q^{2}\right),{ }^{2} A_{l-2}(q)$ \\
${ }^{2} A_{l}(q), l \geq 4, l$ even & end and middle node & ${ }^{2} A_{l-2}(q), A_{(l-2) / 2}\left(q^{2}\right)$ \\
$2 D_{4}(q)$, & end nodes & $A_{3}(q), A_{2}(q)$ \\
${ }^{2} D_{l}(q), l \geq 5$ & end nodes & ${ }^{2} D_{l-1}(q), A_{l-2}(q)$ \\
$E_{6}(q)$ & nodes 1 and 6 & $D_{5}(q)$ \\
$E_{7}(q)$ & nodes 1 and 2 & $D_{6}(q), A_{6}(q)$ \\
$E_{8}(q)$ & nodes 1 and 2 & $D_{7}(q), A_{7}(q)$ \\
$F_{4}(q)$ & end nodes & $B_{3}(q), C_{3}(q)$ \\
$G_{2}(q)$ & end nodes & $A_{1}(q)$ \\
$2 E_{6}(q)$ & end nodes & $2 D_{4}(q),{ }^{2} A_{5}(q)$ \\
\hline
\end{tabular}

The next lemma also eliminates the possibility that $q=3$ for classical groups.

Lemma 12. If $x$ is an element of order 3 in a classical group $G$ defined over $\mathbb{F}_{3}$, then $(x, G)$ cannot be a minimal counterexample.

Proof. The aim is to show that if $x$ is not a long root element, then, unless the dimension of the natural module $V$ is very small, there exists a subgroup $H$ such that $(x, H)$ is in $\mathcal{A}$. By Wal63, pp.34-38], if $x$ is an element of order 3 in a classical group over $\mathbb{F}_{3}$, then $x$ will nearly always fix an orthogonal decomposition unless $n$ is very small. Suppose that $x$ has order 3 in $S L(n, 3)$ with $n \geq 5$. Then there exist $x$-invariant subspaces $U$ and $W$ such that $V=U \oplus W$. Without loss of generality, it suffices to assume that the dimension of $U, k$ say, is at least 3 and $x$ acts nontrivially on $U$. Suppose that $x$ does not act as a transvection on V. If $x$ does not act as a transvection on $U$, then $(x, G)$ cannot be a minimal counterexample. So assume that $x$ acts as a transvection on $U$. Then $x$ must act non-trivially on $W$. So the dimension of $W, n-k$, is at least 2 and it suffices to assume that $x$ acts as a transvection on $W$ also, but since $n \geq 5$ there is a four-dimensional subspace $U^{\prime}$ on which $x$ acts invariantly and is not a transvection and so $x_{U^{\prime}}$ is contained in a subgroup of type $G L(4,3)$. Now suppose that $x$ is contained in a symplectic group $S p(n, 3)$ and that $x$ is not a symplectic transvection. If $n \geq 8$, then $x$ fixes an orthogonal decomposition $U \perp W$. It suffices to assume that $x$ acts non-trivially on both $U$ and $W$; otherwise $(x, G)$ is not a minimal counterexample. Moreover, it suffices to assume that $x$ acts as a symplectic transvection on $U$ and on $W$; otherwise $\left(x_{U}, S p(U)\right)$ or $\left(x_{W}, S p(W)\right)$ is contained in $\mathcal{A}$. So assume that for all $u \in U$ and all $w \in W$,

$$
\begin{aligned}
& x_{U}: u \rightarrow u+\lambda \kappa_{U}(u, a) a, a \in U, \kappa(a, a)=0 ; \\
& x_{W}: w \rightarrow w+\lambda^{\prime} \kappa_{W}(w, b) b, b \in W, \kappa(b, b)=0 .
\end{aligned}
$$


Choose $u \in U$ such that $\kappa_{U}(u, a) \neq 0$ and $w \in W$ such that $\kappa_{W}(w, b) \neq 0$. Then $x$ acts invariantly on the non-degenerate subspace $\langle u, w, a, b\rangle$ and is not a transvection on it, so $(x, G)$ cannot be a minimal counterexample. Now suppose that $x$ is contained in a unitary group $S U(n, 3)$ and that $x$ is not a unitary transvection. Suppose that $n \geq 5$. Then there exists an $x$-invariant orthogonal decomposition $U \perp W$ and, as before, it suffices to assume that $x$ acts non-trivially on both subspaces. Moreover, there exists $H$ such that $(x, H)$ is contained in $\mathcal{A}$ unless $x$ acts as a unitary transvection on both $U$ and $W$. So for all $u \in U$ and all $w \in W$,

$$
\begin{aligned}
& x_{U}: u \rightarrow u+\lambda \kappa_{U}(u, a) a, a \in U, \kappa(a, a)=0 ; \\
& x_{W}: w \rightarrow w+\lambda^{\prime} \kappa_{W}(w, b) b, b \in W, \kappa(b, b)=0 .
\end{aligned}
$$

Choose $u$ and $w$, as in the symplectic case, so that $\langle u, w, a, b\rangle$ is a 4-dimensional, non-degenerate subspace on which $x$ acts invariantly, but not as a transvection. Then $(x, G)$ is not a minimal counterexample in this case either. Finally, suppose that $x$ is contained in an orthogonal group $\Omega^{\epsilon}(n, 3)$ and that $x$ is not a long root element. Suppose that $n \geq 9$. Then there exists an $x$-invariant orthogonal decomposition $U \perp W$. It suffices to assume that the action on $U$ and $W$ is not trivial as in the previous cases. Since $n \geq 9$, it suffices to assume that the dimension of $U, k$ say, is at least 5 . Then if $(x, G)$ is a minimal counterexample, $x$ must act as a long root element on $U$. Now either $x$ acts as a long root element on $W$, or $x$ does not act as a long root element on $W$ and $n-k \leq 4$. In the latter case one can add dimensions from $U$ to $W$ so that $W$ has dimension at least 5 and $W$ is still $x$-invariant and non-degenerate. If this is done, then $x_{W}$ is contained in an orthogonal group $H$ such that $\left(x_{W}, H\right) \in \mathcal{A}$. In the former case, $n-k \geq 4$ ( $W$ has Witt defect 0 if $n-k=4$ ) and for all $u \in U$ and all $w \in W$,

$$
\begin{array}{r}
x_{U}: u \rightarrow u+\lambda \kappa_{U}(u, a) b-\lambda \kappa_{U}(u, b) a, \\
x_{W}: w \rightarrow w+\lambda^{\prime} \kappa_{W}(w, c) d-\lambda^{\prime} \kappa_{W}(w, d) c,
\end{array}
$$

where $a, b \in U ; c, d \in W$; and $Q(a)=Q(b)=\kappa_{U}(a, b)=0=Q(c)=Q(d)=\kappa(c, d)$.

If $u_{1}, u_{2} \in U$ are such that $Q\left(u_{i}\right)=0=\kappa_{U}\left(u_{1}, a\right)=\kappa_{U}\left(u_{2}, b\right)$, and $\kappa_{U}\left(u_{1}, b\right) \neq 0$, $\kappa_{U}\left(u_{2}, a\right) \neq 0$, then $x$ acts invariantly on the non-degenerate 4-dimensional subspace $\left\langle u_{1}, u_{2}, a, b\right\rangle$. Similarly, take $w_{1}, w_{2} \in W$ such that $x$ acts invariantly on the nondegenerate 4-dimensional subspace $\left\langle w_{1}, w_{2}, c, d\right\rangle$. Then $x$ acts invariantly on the non-degenerate 8-dimensional subspace $\left\langle u_{1}, u_{2}, a, b, w_{1}, w_{2}, c, d\right\rangle$, which has Witt defect 0 , and $x$ does not act as a long root element on it. So it is enough to check the classical groups of dimension at most 8 over $\mathbb{F}_{3}$ in MAGMA.

If $x$ is a transvection in $S L(n, 3)$, then one can reduce to the case where $n=3$. Similarly if $x$ is a transvection in $S U(n, 3)$ or $S p(n, 3)$, then one can reduce to the case where $x \in S U(3,3)$ or $x \in S p(4,3)$. If $x$ is a long root element in an orthogonal group, then one can reduce to the six-dimensional case, but $P \Omega^{+}(6,3) \cong P S L(4,3)$ and $x$ maps to a transvection under this isomorphism. We can therefore further reduce to $S L(3,3)$. By GS03, there exist three conjugates of $x$ that generate $G_{0}$ when $G_{0}=P S L(3,3)$ or $P S U(3,3)$, and four conjugates of $x$ that generate $G_{0}=P S p(4,3)$. 


\section{CASE U}

It suffices to assume that $n \geq 3$ and $(n, q) \neq(3,2)$. By Lemmas 711 and 12, if $(x, G)$ is a minimal counterexample, with $G_{0} \cong P S U(n, q)$, then $x$ is a semisimple element in $P G U(n, q)$, or $x$ is unipotent in $P S U(3, q)$, and $q \geq 5$.

9.1. $x \in P G U(n, q), p \nmid q$ and $p \nmid(n, q+1)$. By Lemma 3, $x \in P G U(n, q)$ lifts to an element in $G U(n, q)$ of order $p$, with the same sized conjugacy class. Without loss of generality, it suffices to assume that $G=P G U(n, q)$ by Lemma 4 . Consider the minimal polynomial of $x, m_{x}(t)$ say. Observe that $m_{x}(t)$ divides $t^{p}-1$ and $t^{p}-1 /(t-1)$ factors over $\mathbb{F}_{q^{2}}$ as

$$
q_{1}(t) \ldots q_{s}(t)
$$

where the $q_{i}(x)$ 's are polynomials of degree $k$ (where $k$ is the smallest positive integer such that $\left.p \mid q^{2 k}-1\right)$. The same argument as for the case when $G_{0}=\operatorname{PSL}(n, q)$ shows that $x$ will leave invariant and act non-trivially and irreducibly on a $k$ dimensional subspace $U$ of $V$. Since $x$ acts irreducibly on $U, U$ is either nondegenerate or totally singular (for if $U$ is not non-degenerate, then there exists $v \in U$ such that $\kappa_{U}(v, u)=0$ for all $u \in U$; but $x$ acts irreducibly on $U$ so $\left.\kappa_{U}=0\right)$. If $k \geq 2$, in both cases, consider the induced isometry $x_{U}$ of $U$. If $U$ is totally singular, then $x_{U}$ is contained in a group of type $G L\left(k, q^{2}\right)$ and so $(x, G)$ cannot be a minimal counterexample. If $U$ is non-degenerate, then $x_{U}$ is contained in a subgroup of type $G U(k, q)$. Observe that if $U$ is non-degenerate, then $k$ is odd. For if $k$ were even, then, since $|G U(k, q)|=q^{k(k-1) / 2} \prod_{i=1}^{k}\left(q^{i}-(-1)^{i}\right), p$ would divide $q^{k}-1=q^{2 j}-1$, contradicting the choice of $k$. Thus if $k \geq 2$, then unless $x$ acts irreducibly or $q=2,\left(x_{U}, G U(k, q)\right)$ is contained in $\mathcal{A}$ and $(x, G)$ cannot be a minimal counterexample.

If $q=2$, then there are exceptions in Table 1. If $k>3$, then $x_{U}$ is contained in $G U(k, 2)$, and the assumption on $k$ implies that $p \neq 3$, so $\left(x_{U}, G U(k, 2)\right)$ is contained in $\mathcal{A}$. If $(k, q)=(3,2)$, then $p=7$. Since $U$ is non-degenerate, $x$ also acts invariantly on $U^{\perp}$. For $n \geq 7$, if this action is non-scalar, then $x_{U^{\perp}}$ is contained in $G U(n-3,2)$ and $\left(x_{U^{\perp}}, G U(n-3,2)\right)$ is contained in $\mathcal{A}$. If the action is scalar, then take a non-singular vector $w \in U^{\perp}$, so that $x$ acts invariantly on $U^{\prime}:=U \oplus\langle w\rangle$. Therefore $x_{U^{\prime}}$ is contained in $G U(4,2)$ and, since $p \neq 3$, it follows that $(x, G)$ is not a minimal counterexample.

If $k=1$, then $x$ acts invariantly on a 1-dimensional non-degenerate or singular subspace $U$. Observe that $q \neq 3$ since this would imply that $p=2$. First suppose that $q \neq 2$ so that $q \geq 4$. If $U$ is non-degenerate, then consider the action of $x$ on $U^{\perp}$. Either $x$ acts non-trivially on $U^{\perp}$, in which case $x_{U^{\perp}}$ will be contained in $G U(n-1, q)$, or $x$ has a scalar action on $U^{\perp}$, in which case there exists a 2dimensional non-degenerate subspace $U^{\prime}$ such that $x_{U^{\prime}}$ is contained in $G U(2, q)$. Since $q \geq 4,(x, G)$ cannot be a minimal counterexample in any case. Now suppose that $q=2$ and $k=1$ so that $p=3$. If $x$ has order 3 in $G U(n, 2)$, then a Sylow 3 -subgroup is contained in a subgroup of type $G U(1, q) 2 S_{n}$. So it suffices to assume that $x$ will lie in a subgroup $G U(1,2)<S_{n}$, and if $n \geq 5$, it suffices to assume that $x$ is contained in $G U(1,2) \perp \ldots \perp G U(1,2)$ since otherwise $x$ will be non-trivial in a subgroup of type $S_{n}$. Thus for $n \geq 5$, if $x$ is not a reflection, then there 
exists an $(n-1)$-dimensional, non-degenerate, $x$-invariant subspace $U^{\prime}$ such that $x_{U^{\prime}} \in G U(n-1,2)$ with $\left(x_{U^{\prime}}, G U(n-1,2)\right)$ contained in $\mathcal{A}$. A MAGMA calculation shows that the only exceptions to the theorem for $G:=P G U(4,2)$ are reflections of order 3. If $x$ is a reflection of order 3 in $G U(n, 2)$, then it suffices to treat the case where $x$ is contained in $G U(4,2)$. A calculation in MAGMA shows that there exist $g_{1}, g_{2}, g_{3} \in G$ such that $\left\langle x, x^{g_{1}}, x^{g_{2}}, x^{g_{3}}\right\rangle$ is not solvable.

If $k=1$ and there is not a 1 -dimensional non-degenerate $x$-invariant subspace, then $U$ is totally singular and $x$ is contained in a parabolic subgroup. Thus by Lemma 6, it suffices to assume that $x$ acts non-centrally on each component of the Levi complement of some maximal parabolic subgroup. The parabolic subgroups of ${ }^{2} A_{m}(q)$ have Levi complements of type ${ }^{2} A_{m-2}(q), A_{k}\left(q^{2}\right)^{2} A_{m-2 k-2}(q)$ and, if $m$ is odd, ${ }^{2} A_{(m-1) / 2}\left(q^{2}\right)$. So $(x, G)$ cannot be a minimal counterexample unless $m=2$, $(m, q)=(3,2)$, or $(m, q)=(4,2)$. If $m=2$, then $x$ is a reducible semisimple element in $G U(3, q)$, so $q \geq 4$ and so $x$ leaves invariant a 2-dimensional, non-degenerate subspace $U^{\prime}$. In this case, $x_{U^{\prime}}$ is contained in $G U(2, q)$ and so $\left(x_{U^{\prime}}, G U(2, q)\right)$ is contained in $\mathcal{A}$. When $(m, q)=(3,2), G_{0}=P S U(4,2)$. When $(m, q)=(4,2)$, $G_{0} \cong P S U(5,2)$. These cases can be excluded using MAGMA.

The remaining case occurs when $k=n$ and $x$ acts irreducibly in $G U(n, q)$, where $n \geq 3$ is odd (and $(n, q) \neq(3,2))$. Now one can use GPPS99 to find the possibilities for a maximal subgroup $M$ containing $\left\langle x, x^{g}\right\rangle$ in $G U(n, q)$. Note that $x$ is a $\operatorname{ppd}\left(n, q^{2}, n\right)$-element, and that $n \geq 3$ is odd. So since $n$ is not a power of 2 there are no 2.5 examples. Lemma 9 implies that $M$ must be a type 2.1 or $2.4(\mathrm{~b})$ subgroup. By [KL90], the only possible such classical maximal subgroups are of type $G U\left(n, q_{0}\right)$ and $O_{n}(q)$ ( $q$ odd). The only subgroups of this type that contain an element of order $p \geq 5$ and are not almost simple modulo scalars are those of type $O_{3}(3)$ when $n=q=3$. One can treat $G U(3,3)$ separately in MAGMA. The only other examples are the field extension examples (type 2.4(b)). By [KL90] and since $n$ is odd these are subgroups of type $G U\left(n / r, q^{r}\right)$, where $r$ is an odd prime. Now unless $n=r$ these subgroups are almost simple modulo scalars and thus $(x, M)$ is contained in $\mathcal{A}$. If $n=r$ and $x \in M$, where $M$ is a subgroup of type $G U\left(1, q^{n}\right)$, then observe that $x$ is contained in only one such maximal subgroup and Theorem 4 implies that $(x, G)$ cannot be a minimal counterexample.

9.2. $x \in P G U(n, q)$ and $p \mid(q+1, n)$. Observe that Lemma 4 still applies, so assume that $G=P G U(n, q)$. This time some conjugacy classes of order $p$ could only lift to non-trivial scalars in $G U(n, q)$. If $x$ lifts to an element of order $p$ in $G U(n, q)$, then apply the same argument as in the previous section. If not, then $x^{p}$ lifts to a non-trivial scalar in $G U(n, q)$. So $x$ will have order $p^{m} j$ say, where $p \nmid j$, but since $\left\langle x^{j}\right\rangle \leq\langle x\rangle$ and $x^{j}$ will still have order $p$ in $P G U(n, q)$, it suffices to assume that $j=1$. So assume that the order of $x$ in $G U(n, q)$ is $p^{m}$. The minimal polynomial of $x, m_{x}(t)$, divides $t^{p}-\zeta_{p^{m-1}}$, where $\zeta_{p^{m-1}}$ is a primitive $p^{m-1}$ th root of unity in $\mathbb{F}_{q^{2}}$, and $p^{m-1} \mid(q+1)$. Since there are $p$ th roots of unity, either $t^{p}-\zeta_{p^{m-1}}$ splits, or it is irreducible over $\mathbb{F}_{q^{2}}$. For if $a$ is a root of the equation $t^{p}-\zeta_{p^{m-1}}=0$ contained in some field extension, then this field extension contains all of the roots, $a \omega, a \omega^{2}, \ldots, a \omega^{p-1}$. So $t^{p}-\zeta_{p^{m-1}}$ will factor into irreducible polynomials of degree equal to the degree of the smallest field extension containing $a$. However, $p$ is prime, so the degree of these polynomials is either 1 or 
$p$. If $t^{p}-\zeta_{p^{m-1}}$ splits, then $m_{x}(t) \mid\left(t-\zeta_{p^{m}}\right)\left(t-\zeta_{p^{m}} \omega\right) \ldots\left(t-\zeta_{p^{m}} \omega^{p-1}\right)$, where $\zeta_{p^{m}}$ is a primitive $p^{m}$ th root of unity in $\mathbb{F}_{q^{2}}$. However this would imply that $p^{m}$ divides $q+1$. For $p^{m-1} \mid(q+1)$, and since $\zeta_{p^{m}} \in \mathbb{F}_{q^{2}}, p^{m} \mid(q-1)(q+1)$, but $p \nmid q-1$ since $p \geq 3$. This would be a contradiction, since $z=\zeta_{p^{m}} I_{n}$ would lie in $Z(G U(n, q))$, so $\left(z^{-1} x\right)^{p}=\zeta_{p^{m-1}}^{-1} \zeta_{p^{m-1}} I_{n}=I_{n}$, and $x$ would lift to an element of order $p$. So, it suffices to assume that $m_{x}(t)=t^{p}-\zeta_{p^{m-1}}$ is irreducible over $\mathbb{F}_{q^{2}}$. It follows that $x$ has rational canonical form $\operatorname{diag}\left[A_{1}, \ldots, A_{n / p}\right]$, where

$$
A_{i}=\left(\begin{array}{ll}
\zeta_{p-1} \\
\zeta_{p^{m-1}}
\end{array}\right)
$$

Thus $x$ acts irreducibly on a subspace $W$ of dimension $p$. Now $p^{m} \mid q^{2 p}-1$, and in fact, $p^{m} \mid q^{p}+1$, since if $p \mid q^{p}-1$, then $q^{p} \equiv 1(\bmod p)$ but also $q^{p} \equiv q(\bmod p)$ by Fermat's Little Theorem. Therefore $q \equiv 1(\bmod p)$, and $p \mid q+1$, which contradicts the assumption that $p \geq 3$. So $p^{m}$ divides $q^{p}+1$.

Assume that $W$ is non-degenerate since if $W$ were totally singular, then $x_{W}$ would be contained in $G L\left(p, q^{2}\right)$ and $\left(x_{W}, G L\left(p, q^{2}\right)\right)$ would be contained in $\mathcal{A}$. Thus, if $x$ does not lift to an element of order $p$, then it suffices to assume that $n=p$ and that $x$ acts irreducibly.

Since $n=p$, and $p \mid q+1$, one can show that a maximal subgroup $M$ of $G U(p, q)$ of type $G U(1, q) \gtrless S_{p}$ always contains a Sylow $p$-subgroup of $G U(p, q)$. Thus, it suffices to assume that $x$ is contained in $M$, the normalizer of a maximal split torus $T$. Moreover, $x$ is non-trivial in $N_{G}(T) / T \cong S_{p}$, since it acts irreducibly. So if $p \geq 5$, then $x$ cannot be a minimal counterexample. Now suppose that $p=3$. Then $x$ is an irreducible element in $G U(3, q)$. The character table of $G U(3, q)$ in Enn62 with the same argument as when $G_{0}=P S L(2, q)$ implies that there exists an element $z$ in $G U(3, q)$ of order $q^{2}-1$ such that $x$ is conjugate to $x z$. So, if $x^{g}=x z$, then $\left\langle x, x^{g}\right\rangle=\langle x, z\rangle$ contains $\operatorname{PSU}(3, q)$, since it cannot be contained in any of the maximal subgroups described in GLS98, Theorem 6.5.3].

9.3. $x \in P S U(3, q)$ and $p \mid q, q \geq 5$. If $x$ is a unipotent element in $G_{0}=P S U(3, q)$, then the maximal subgroups of $G_{0}$ are described in [GLS98, Theorem 6.5.3] and Lemma 5 can be applied. By Lemma [12, there are no minimal counterexamples when $q=3$. So assume that $q \geq 5$. If $x$ is a transvection, then it stabilizes a nondegenerate 2-dimensional subspace, and acts non-trivially on it, so $(x, G)$ cannot be a minimal counterexample. Thus $x$ is not a transvection and $\left|C_{P S U(3, q)}(x)\right|=$ $q^{2}$. Since $(x, G)$ is a minimal counterexample, the only possibilities for maximal subgroups $X_{i}$ containing $x$ are of type $G U(1, q)$ 乙 $S_{3}$ (for $p=3$ ), $G U\left(1, q^{3}\right.$ ) (for $p=3)$, and parabolic subgroups. Note that $P S U(3,2)$ and $P G U(3,2)$ do not contain $x$ since they are $\{2,3\}$-groups that are only relevant when $3 \nmid q$. There is only one conjugacy class of each of the given subgroups and $\left|x^{P S U(3, q)} \cap X_{i}\right|$ is at most $6(q+1)^{2}, 3\left(q^{2}-q+1\right)$, and $q^{3}-1$ in each case respectively. So

$$
\begin{aligned}
& |G| /\left|C_{G}(x)\right|^{2}=q^{3}\left(q^{2}-1\right)\left(q^{3}+1\right) / q^{4}=\left(q^{2}-1\right)\left(q^{3}+1\right) / q \\
& \geq\left(q^{3}-1\right)+\left(q^{2}-q+1\right) \cdot 3+(q+1)^{2} .6 \geq \sum_{i}\left|x^{G} \cap X_{i}\right|
\end{aligned}
$$

for $q \geq 5$, and thus $(x, G)$ cannot be a minimal counterexample by Lemma 5 , 


\section{CAse S}

If $G_{0} \cong P S p(n, q)$, then the only case left to prove occurs when $x$ is a semisimple element contained in $\operatorname{Inndiag}(P S p(n, q)) \cong P G S p(n, q)$. Since $\mid P G S p(n, q)$ : $P S p(n, q) \mid=(2, q-1), x$ must be contained in $P S p(n, q)$, so suppose that $G=G_{0}$. Furthermore, by Lemma [3, $x$ always lifts to an element in $S p(n, q)$ of order $p$.

Let $e$ be the smallest positive integer such that $p \mid q^{e}-1$. Hence the minimal polynomial of $x$ will be a product of irreducibles of degree $e$, and possibly $t-1$. Also, $V$ will have an $e$-dimensional $x$-invariant subspace $U$, on which $x$ acts irreducibly. $U$ is either totally singular or non-degenerate. This depends on $e$ :

- $e$ odd and $e \neq 1$. If $e$ is odd, then $U$ is totally singular since there are no non-degenerate subspaces of $V$ of odd order. So, if $e \geq 3$, then it suffices to assume that $x$ acts non-trivially on $U$, and $x_{U}$ is contained in a subgroup $H$ of type $G L(e, q)$. Clearly $(x, H)$ is contained in $\mathcal{A}$ in this case.

- $e=1$. If $e$ is 1 , then $U$ is a 1-dimensional totally singular subspace, so $x$ is contained in a parabolic subgroup. By Lemma 6, it suffices to assume that $x$ acts non-centrally on all the components of the Levi complement of a maximal parabolic subgroup. This maximal parabolic subgroup can be of type $C_{m-1}(q)(m \geq 3) ; A_{k}(q) C_{m-k-1}(q)(m \geq 4,1 \leq k \leq m-3)$; $A_{m-1}(q)$; or $A_{1}(q) A_{1}(q)(m=3)$. Since $p \mid q-1, q$ is at least 4 ; thus $(x, G)$ cannot be a minimal counterexample.

- e even, $e<n$. If $U$ is totally singular, then $x_{U}$ is contained in a subgroup $H$ of type $G L(e, q)$, and $(x, H)$ is in $\mathcal{A}$ unless $(e, q)=(2,2)$ (if $(e, q)=(2,3)$, then $p=2)$. If $(e, q)=(2,2)$, then it suffices to assume that $n \geq 8$, since $S p(4,2) \cong S_{6}$, and the case $S p(6,2)$ can be excluded using MAGMA. Since $U$ is totally singular, $x$ is contained in a parabolic subgroup, so we can use Lemma 6 as in the previous case. It follows that $(x, G)$ cannot be a minimal counterexample in this case either. If $U$ is non-degenerate, then $x_{U}$ is contained in a subgroup $H$ of type $S p(e, q) .(x, H)$ is contained in $\mathcal{A}$ for $e \geq 4$ and for $e=2, q \geq 4$. If $e=2$, and $q \leq 3$, then $q=2$. But it suffices to assume that $n \geq 6$, since $S p(4,2) \cong S_{6}$, so $x_{U^{\perp}}$ is contained in a subgroup $H$ of type $S p(n-e, 2)$ and $(x, H)$ is contained in $\mathcal{A}$.

If $x$ acts irreducibly, then GPPS99 describes the possible maximal subgroups of $S p(n, q)$ that could contain $x$. It suffices to assume that $n$ is at least 4 , since $S L(2, q) \cong S p(2, q)$. The only $M$ 's of concern are those that contain $\operatorname{ppd}(n, q, n)$ elements. By Lemma 9, it suffices to assume that $M$ is a subgroup of type 2.1, 2.4(b) or 2.5. If $M$ were a subgroup of type 2.1 , then so long as $M$ is almost simple modulo scalars, $(x, M)$ is contained in $\mathcal{A}$. By [KL90, the only possible such maximal subgroups $M$ are type $2.1(\mathrm{~b})$, where $M$ contains $S p\left(n, q_{0}\right)$; and type $2.1(\mathrm{~d})$, where $M$ contains $\Omega^{\epsilon}\left(n, q_{0}\right)$ for $q_{0}$ even. In these cases, $M$ is almost simple and $(x, M)$ is contained in $\mathcal{A}$ unless $(n, q)=(4,2)$. However since $S p(4,2) \cong S_{6}$, this case can be excluded. If $M$ is of type 2.5, then by [KL90], $M$ would be of type $P . O^{-}(2 m, 2)$, where $q$ is an odd prime, $n=2^{m}$, and $P$ is a 2 -subgroup. However since $x$ has odd order, $x O_{2}(M)$ would be non-trivial in the quotient $M / O_{2}(M)$. Moreover, $e \geq 4$ implies that $m \geq 2$ and thus $M / O_{2}(M)$ is almost simple of type $O^{-}(2 m, 2)$. The only other possibility for $M$ is to be of type $2.4(\mathrm{~b})$. In this case, 
by [KL90], $M$ would be of type $S p\left(n / b, q^{b}\right)$, where $b$ is a prime and $n / b$ is even; or of type $G U(n / 2, q)$. However, since $n \geq 4$, these are all almost simple modulo scalars unless $(n, q)=(4,2),(4,3)$, or $(6,2)$. These exceptions are not a problem since $S p(4,2) \cong S_{6}, P S p(4,3) \cong P S U(4,2)(p \neq 3$ since $x$ is a $\operatorname{ppd}(4,3,4)$-element $)$ and there are no elements of prime order in $S p(6,2)$ that act irreducibly.

\section{Case O}

It suffices to assume that $n \geq 7$ since otherwise $G_{0}$ is isomorphic to one of the classical groups that have already been considered. If $x \in \operatorname{Inndiag}\left(P \Omega_{n}^{\epsilon}(q)\right)$ has odd prime order, then $x \in P \Omega_{n}^{\epsilon}(q)$. By Lemma 3, $x$ lifts to an element of order $p$ in $\Omega_{n}^{\epsilon}(q)$. Lemmas 17, 11] and 12 imply that if $(x, G)$ is a minimal counterexample, then $x \in \operatorname{Inndiag}\left(G_{0}\right)$ and $x$ is semisimple.

Let $e$ be minimal such that $p \mid q^{e}-1$, so there exists an $e$-dimensional subspace $U$ on which $x$ acts invariantly and irreducibly. Consider the different values for $e$ :

- $e$ odd, $e \geq 3$. If $e$ is odd, then $p \nmid|O(e, q)|$, so $U$ must be totally singular. It follows that $x_{U}$ is contained in a subgroup $H$ of type $G L(e, q)$ and $\left(x_{U}, H\right) \in$ $\mathcal{A}$.

- $e=1$. If $e=1$, then $q \geq 4$ since $p \mid q-1$. If $x$ acts invariantly on a non-degenerate 1-dimensional subspace $U$, then consider the action of $x$ on $U^{\perp}$. If this action is non-scalar, then $(x, G)$ is not a minimal counterexample since $x_{U^{\perp}}$ is contained in a subgroup $H$ of type $O^{\epsilon}(n-1, q)$ and $\left(x_{U^{\perp}}, H\right)$ is contained in $\mathcal{A}$ since $n \geq 7$. If the action is scalar, then there exists a 3-dimensional subspace $Y$ of $U^{\perp}$ such that $U^{\prime}:=U \oplus Y$ is nondegenerate and $x$-invariant. In this case, $x_{U^{\prime}}$ will be contained in a subgroup $H$ of type $O^{\epsilon}(4, q)$. In particular, $\left(x_{U^{\prime}}, H\right)$ would be contained in $\mathcal{A}$. If $x$ acts invariantly on a singular, 1-dimensional subspace, then $x$ is contained in a parabolic subgroup. Thus, by Lemma 6, it suffices to assume that $x$ acts non-centrally on each component of the Levi complement of some maximal parabolic subgroup. The possible types of maximal parabolic subgroups are: $A_{m-1}(q)$, or $B_{m-1}(q)$ if $G_{0}=B_{m}(q) ; D_{m-1}(q), A_{m-1}(q)$, $A_{m-3}(q) A_{1}(q) A_{1}(q)$, or $A_{k}(q) D_{m-k-1}(q)$ if $G_{0}=D_{m}(q)$; or ${ }^{2} D_{m-1}(q)$, $A_{m-2}(q), A_{k}(q)^{2} D_{m-k-1}(q)$, or $A_{m-3}(q) A_{1}\left(q^{2}\right)$ if $G_{0}={ }^{2} D_{m}(q)$. Since if $G_{0}=B_{m}(q)$, then $m \geq 3$ and in the other cases $m \geq 4$, it follows that $(x, G)$ cannot be a minimal counterexample.

- $e=2$. If $e=2$, then $p \mid q+1$. If $U$ is totally singular, then $x$ is contained in a parabolic subgroup and Lemma 6 is applied as above. If $G_{0}=B_{m}(q)$, then $m \geq 3$ and $q \geq 5$ since $B_{m}\left(2^{a}\right) \cong C_{m}\left(2^{a}\right)$. The only complication is that if $G_{0}=D_{4}(2)$, then all of the components of a parabolic subgroup of type $A_{1}(q) A_{1}(q) A_{1}(q)$ are solvable. One can verify in MAGMA that there are no counterexamples when $G_{0}=D_{4}(2)$. Now suppose that $x$ acts invariantly on a 2-dimensional non-degenerate subspace $U$. Then $U$ will be anisotropic because of the order of $x$. If the action of $x$ on $U^{\perp}$ is nonscalar, then $x_{U^{\perp}}$ will be contained in a subgroup $H$ of type $O^{-\epsilon}(n-2, q)$ (since $U$ has Witt defect 1, [KL90, 4.1.6]) and $\left(x_{U^{\perp}}, H\right)$ will be contained in $\mathcal{A}$. Suppose that $x$ acts as a scalar on $U^{\perp}$. In this case, let $W$ be a 4-dimensional non-degenerate subspace of $U^{\perp}$ (of Witt defect 0 ). Then $x$ will act invariantly on the non-degenerate space $U^{\prime}=U \oplus W$. So $x_{U^{\prime}}$ will be 
contained in a subgroup $H$ of type $O^{-}(6, q)$, and $\left(x_{U^{\prime}}, H\right)$ will be contained in $\mathcal{A}$.

- $e$ even, $e \geq 4$. If $e$ is even, then $p \mid q^{e / 2}+1$. Suppose that $U$ is totally singular. Then $x_{U}$ will be contained in a subgroup $H$ of type $G L(e, q)$, and $\left(x_{U}, H\right)$ will be contained in $\mathcal{A}$ since $e \geq 4$. So assume that $U$ is nondegenerate. If $e \neq n$, then $x_{U}$ will lie in a subgroup $H$ of type $O^{-}(e, q)$, with $\left(x_{U}, H\right)$ contained in $\mathcal{A}$. The only case left to consider is where $x$ acts irreducibly on $O^{-}(e, q)$.

Since $e=n$ is even it suffices to assume that $n \geq 8$. One can use KL90 and GPPS99 to find the possible maximal overgroups of $x$. Lemma 9 implies that $M$ must be a subgroup of type 2.1, 2.4(b) or 2.5. The only subgroups $M$ of type 2.1 are of type $O^{-}\left(n, q_{0}\right)$, and if $M$ were such a subgroup, then $(x, M)$ would be contained in $\mathcal{A}$. There are no symplectic type normalizer maximal subgroups in $O^{-}(n, q)$, so there are no 2.5 type maximal subgroups. This leaves field extension examples of type 2.4. The possibilities are subgroups of type $G U(n / 2, q), O^{-}\left(n / 2, q^{2}\right)$, and $O^{-}\left(n / r, q^{r}\right)$ for $r$ a prime and $e / r \geq 4$. All of these are almost simple modulo scalars and $(x, M)$ would be contained in $\mathcal{A}$. Thus, $(x, G)$ cannot be a minimal counterexample.

\section{2. $E_{l}(q)$}

Now suppose that $G_{0}$ is an exceptional group of type $E_{l}(q)$, for $l=6,7$, or 8 . If $(x, G)$ is a minimal counterexample, then by Lemmas 7 and 11 either $x \in G_{0}$ and $p=q=3$, or $x \in \operatorname{Inndiag}\left(G_{0}\right)$ and $p \nmid q$.

First suppose that $p=q=3$. If $x$ is a long root element, then $\left\langle x, x^{g}\right\rangle$ is either a 3-group or a fundamental $S L(2,3)$ subgroup, by [GLS98, Proposition 3.2.9]. The unipotent conjugacy classes are described in [Miz77, Miz80. Tables 4, 5, and 6] list the representatives for the unipotent classes of order 3 in $E_{l}(3)$ and describe a subsystem subgroup $H$ containing each representative. The tables show that there are no minimal counterexamples when $x$ is unipotent. 


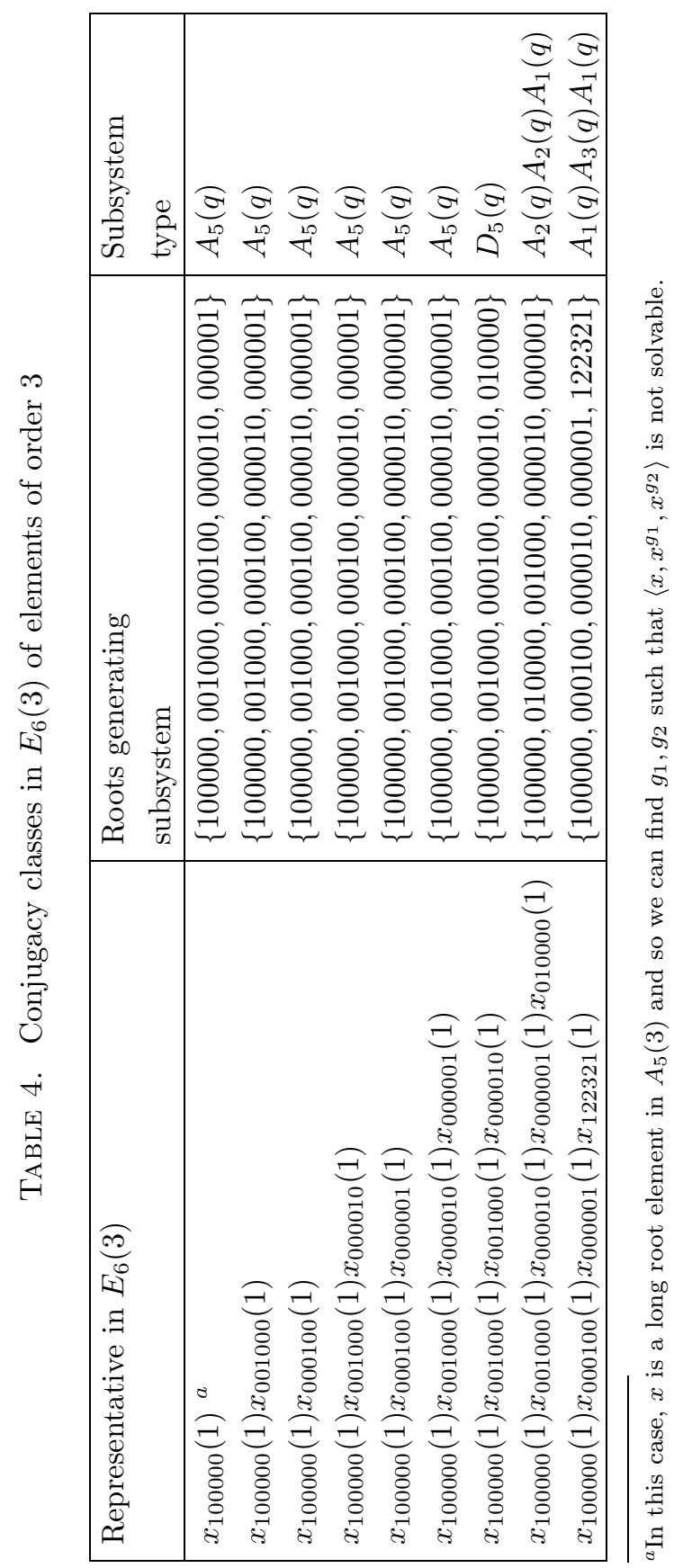


TABLE 5. Conjugacy classes in $E_{7}(3)$ of elements of order 3

\begin{tabular}{|l|l|l|}
\hline Representative in $E_{7}(3)$ & $\begin{array}{l}\text { Roots generating } \\
\text { subsystem }\end{array}$ & $\begin{array}{l}\text { Subsystem } \\
\text { type }\end{array}$ \\
\hline$x_{34}(1) x_{36}(1) x_{37}(1) x_{38}(1) x_{40}(1)$ & $\alpha_{34}, \alpha_{40}, \alpha_{36}, \alpha_{38}, \alpha_{37}$ & $A_{2}(q) A_{2}(q) A_{1}(q)$ \\
$x_{34}(1) x_{36}(1) x_{38}(1) x_{40}(1)$ & $\alpha_{34}, \alpha_{40}, \alpha_{36}, \alpha_{38}$ & $A_{2}(q) A_{2}(q)$ \\
$x_{37}(1) x_{38}(1) x_{39}(1) x_{40}(1) x_{41}(1)$ & $\alpha_{37}, \alpha_{38}, \alpha_{39}, \alpha_{40}, \alpha_{41}$ & $A_{1}(q)^{2} A_{2}(q) A_{1}(q)$ \\
$x_{42}(1) x_{43}(1) x_{44}(1) x_{45}(1)$ & $\alpha_{42}, \alpha_{45}, \alpha_{43}, \alpha_{44}$ & $A_{2}(q) A_{1}(q) A_{1}(q)$ \\
$x_{44}(1) x_{46}(1) x_{49}(1)$ & $\alpha_{44}, \alpha_{46}, \alpha_{49}$ & $A_{2}(q) A_{1}(q)$ \\
$x_{42}(1) x_{43}(1) x_{44}(1) x_{51}(\zeta) x_{49}(1)$ & $\alpha_{3}, \alpha_{5}, \alpha_{7}, \alpha_{38}, \alpha_{49}$ & $D_{4}(q) A_{1}(q)$ \\
$x_{44}(1) x_{46}(1)$ & $\alpha_{44}, \alpha_{46}$ & $A_{2}(q)$ \\
$x_{42}(1) x_{43}(1) x_{44}(1) x_{51}(\zeta)$ & $\alpha_{3}, \alpha_{5}, \alpha_{7}, \alpha_{38}$ & $D_{4}(q)$ \\
$x_{47}(1) x_{48}(1) x_{49}(1) x_{53}(1)$ & $\alpha_{3}, \alpha_{5}, \alpha_{44}, \alpha_{53}, \alpha_{49}$ & $A_{3}(q) A_{1}(q) A_{1}(q)$ \\
$x_{47}(\zeta) x_{48}(1) x_{49}(1) x_{53}(1)$ & $\alpha_{3}, \alpha_{5}, \alpha_{44}, \alpha_{53}, \alpha_{49}$ & $A_{3}(q) A_{1}(q) A_{1}(q)$ \\
$x_{47}(1) x_{48}(1) x_{49}(1)$ & $\alpha_{3}, \alpha_{5}, \alpha_{44}, \alpha_{49}$ & $A_{3}(q) A_{1}(q)$ \\
$x_{47}(\zeta) x_{48}(1) x_{49}(1)$ & $\alpha_{3}, \alpha_{5}, \alpha_{44}, \alpha_{49}$ & $A_{3}(q) A_{1}(q)$ \\
$x_{53}(1) x_{54}(1) x_{55}(1)$ & $\alpha_{2}, \alpha_{7}, \alpha_{50}, \alpha_{55}$ & $A_{3}(q) A_{1}(q)$ \\
$x_{58}(1) x_{59}(1)$ & $\alpha_{2}, \alpha_{5}, \alpha_{57}$ & $A_{3}(q)$ \\
$x_{63}(1) a$ & $\alpha_{1}, \alpha_{62}$ & $A_{2}(q)$ \\
\hline
\end{tabular}

${ }^{a}$ In this case, $x$ is a long root element in $A_{2}(3)$ and so we can find $g_{1}, g_{2}$ such that $\left\langle x, x^{g_{1}}, x^{g_{2}}\right\rangle$ is not solvable. 


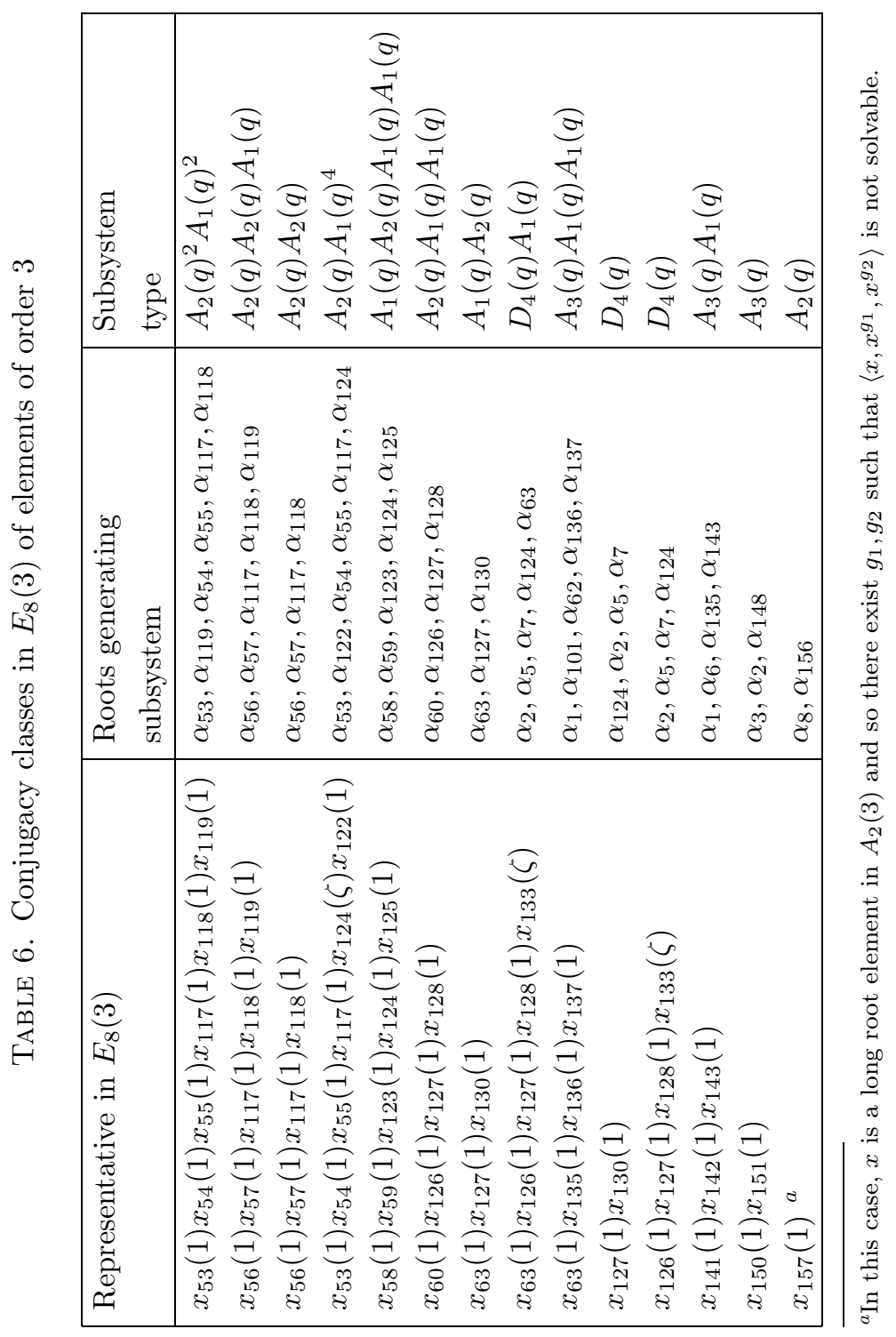


TABle 7. Bounds on $\left|x^{G} \cap X_{i}\right|$ for subgroups $X_{i}$ of $E_{6}(q), p \geq 5$. $d=(2, q-1), e=(3, q-1), f=(3, q+1), g=\left(3, q^{2}-1\right)$, $h=(4, q-1)$

\begin{tabular}{|l|l|l|}
\hline$X_{i}$ & Bound on $\left|x^{G} \cap X_{i}\right|$ & Cruder bound \\
\hline$d .(L(2, q) \times L(6, q)) \cdot d e$ & 0 & 0 \\
$e . L(3, q)^{3} \cdot e^{2} \cdot S_{3}$ & 0 & $q^{9}$ \\
$f .\left(L\left(3, q^{2}\right) \times U(3, q)\right) \cdot g \cdot 2$ & 0 & 0 \\
$L\left(3, q^{3}\right) \cdot(e \times 3)$ & 0 & 0 \\
$d^{2} \cdot\left(P \Omega^{+}(8, q) \times(q-1 / d)^{2}\right) \cdot d^{2} \cdot S_{3}$ & $q^{2}$ & \\
$\left({ }^{3} D_{4}(q) \times\left(q^{2}+q+1\right)\right) \cdot 3$ & $q^{2}+q+1$ & $q^{3}$ \\
$h \cdot\left(P \Omega^{+}(10, q) \times(q-1 / h)\right) \cdot h$ & $h(q-1)$ & $q^{3}$ \\
$(q-1)^{6} \cdot W\left(E_{6}\right), q \geq 5$ & $(q-1)^{6} .51840$ & $q^{13}$ \\
$\left(q^{2}+q+1\right)^{3} \cdot 3^{1+2} S L(2,3)$ & $\left(q^{2}+q+1\right)^{3}$ & $q^{9}$ \\
$3^{3+3} \cdot S L(3,3)$ & 0 & \\
\hline
\end{tabular}

The only other possibility is that $p \nmid q$ and $x \in \operatorname{Inndiag}\left(G_{0}\right)$. By Lemma 4, it suffices to assume that $G=\operatorname{Inndiag}\left(G_{0}\right)$. If $x$ is semisimple, then consider the case where $x$ is contained in a parabolic subgroup. By Lemma 6, there is a conjugate of $x$ that is contained in a maximal parabolic subgroup that does not centralize any component of the Levi complement. For $l=6, P$ will be of type $D_{5}(q), A_{1}(q) A_{4}(q)$, or $A_{5}(q)$, and so $(x, G)$ cannot be a minimal counterexample. Similarly, for $l=7$ and 8 one can reduce to a case where $x$ is acting non-centrally on a component of a Levi complement. So it suffices to assume that $x$ is not contained in any parabolic subgroups. In this case, $x$ is semisimple, and $C_{G}(x)$ is a reductive group containing no unipotent elements. Thus, $C_{G}(x)$ is a torus, and by Sei83, for example, it follows that $\left|C_{G}(x)\right| \leq(q+1)^{l}$. The conjugacy classes of semisimple elements of order 3 are described in [GLS98, Table 4.7.3A]. So if $x$ is not contained in a parabolic subgroup, then it suffices to assume that $p \geq 5$, since $\left|C_{G}(x)\right|>(q+1)^{l}$ for any $x \in E_{l}(q)$ of order 3 . This observation is useful since it implies that $x \in O_{\infty}(M)$ for all maximal subgroups $M$ containing $x$; otherwise $(x, G)$ could not be a minimal counterexample. If $l=6$, then

$$
|G| /\left|C_{G}(x)\right|^{2} \geq \frac{q^{36}\left(q^{12}-1\right)\left(q^{9}-1\right)\left(q^{8}-1\right)\left(q^{6}-1\right)\left(q^{5}-1\right)\left(q^{2}-1\right)}{3(q+1)^{12}},
$$

which is at least $q^{55}$, for $q \geq 2$. The maximal subgroups of $E_{6}(q)$ are described in LS03 and LSS92. The possible maximal subgroups $X_{i}$ containing $x$ are listed in Table 7 together with a crude bound on $\left|x^{G} \cap X_{i}\right|$. Clearly the hypotheses of Lemma 5 are satisfied and there is no minimal counterexample when $l=6$.

Now suppose that $l=7$. Then

$$
\frac{|G|}{\left|C_{G}(x)\right|^{2}} \geq \frac{q^{63}\left(q^{18}-1\right)\left(q^{14}-1\right)\left(q^{12}-1\right)\left(q^{10}-1\right)\left(q^{8}-1\right)\left(q^{6}-1\right)\left(q^{2}-1\right)}{2(q+1)^{14}},
$$

which is at least $q^{111}$ for $q \geq 2$. Table 8 implies that the hypotheses of Lemma 5 are satisfied, and there is no minimal counterexample when $l=7$. If $l=8$, then

$\frac{|G|}{\left|C_{G}(x)\right|^{2}} \geq \frac{q^{120}\left(q^{30}-1\right)\left(q^{24}-1\right)\left(q^{20}-1\right)\left(q^{18}-1\right)\left(q^{14}-1\right)\left(q^{12}-1\right)\left(q^{8}-1\right)\left(q^{2}-1\right)}{2(q+1)^{16}}$,

which is at least $q^{239}$ for $q \geq 2$. Table 9 shows that the hypotheses of Lemma 5 are 
TABle 8. Bounds on $\left|x^{G} \cap X_{i}\right|$ for subgroups $X_{i}$ of $E_{7}(q), p \geq 5$. $d=(2, q-1), e=(3, q-\epsilon), f=(4, q-\epsilon) / d, g=(8, q-\epsilon) / d$

\begin{tabular}{|l|l|}
\hline$X_{i} \leq E_{7}(q)$ & Bound on $\left|x^{G} \cap X_{i}\right|$ \\
\hline$d .\left(L(2, q) \times P \Omega^{+}(12, q)\right) \cdot d$ & 0 \\
$f . L^{\epsilon}(8, q) \cdot g \cdot(2 \times(2 / f), \epsilon=+1$ & 0 \\
$f . L^{\epsilon}(8, q) \cdot g \cdot(2 \times(2 / f), \epsilon=-1$ & 0 \\
$e \cdot L^{\epsilon}(3, q) \times L^{\epsilon}(6, q) \cdot d e \cdot 2, \epsilon=+1$ & 0 \\
$e . L^{\epsilon}(3, q) \times L^{\epsilon}(6, q) \cdot d e \cdot 2, \epsilon=-1$ & 0 \\
$d^{2} \cdot\left(L(2, q)^{3} \times P \Omega^{+}(8, q)\right) \cdot d^{3} \cdot S_{3}$ & 0 \\
$\left(L\left(2, q^{3}\right) \times{ }^{3} D_{4}(q)\right) .3 d$ & 0 \\
$d^{3} \cdot\left(L(2, q)^{7} \cdot d^{4} \cdot L(3,2)\right)$ & 0 \\
$L\left(2, q^{7}\right) \cdot 7 d$ & 0 \\
$e \cdot\left(E_{6}(q) \times(q-1) / e\right) \cdot e \cdot 2, \epsilon=1$ & $q$ \\
$e .\left({ }^{2} E_{6}(q) \times(q+1) / e\right) \cdot e \cdot 2, \epsilon=-1$ & $q^{2}$ \\
$(q-1)^{7} \cdot W\left(E_{7}\right)$ & $q^{30}$ \\
$(q+1)^{7} \cdot W\left(E_{7}\right)$ & $q^{37}$ \\
$\left(2^{2} \times P \Omega^{+}(8, q) \cdot 2^{2}\right) \cdot S_{3}$ & 0 \\
${ }^{3} D_{4}(q) \cdot 3$ & 0 \\
\hline
\end{tabular}

satisfied, and $(x, G)$ cannot be a minimal counterexample.

$$
\text { 13. }{ }^{2} E_{6}(q)
$$

If $x$ is unipotent, then Lemma 11 implies that $p=3$. For $q=3$, the unipotent class representatives were obtained from Frank Lübeck, using CHEVIE (GHL96]). From the class representatives, one can deduce that $(x, G)$ cannot be a minimal counterexample. If $x$ is semisimple, and contained in a maximal parabolic subgroup, then, by Lemma 6, it suffices to assume that $x$ acts non-centrally on all of the components of the Levi complement. If this parabolic subgroup is an end-node parabolic subgroup, then the Levi complement is of type ${ }^{2} D_{4}(q)$ or ${ }^{2} A_{5}(q)$. If $P$ is not an end-node parabolic subgroup, then it can be either of type $A_{1}\left(q^{2}\right) A_{2}(q)$ or $A_{1}(q) A_{2}\left(q^{2}\right)$. Thus, $(x, G)$ cannot be a minimal counterexample if $x$ is contained in a parabolic subgroup.

So suppose that $x$ is semisimple and does not lie in any parabolic subgroups. Then $C_{G}(x)$ is a torus, and as in the previous section, note that if $x$ has order 3, then $\left|C_{G}(x)\right|>(q+1)^{6}$ (by [GLS98, Table 4.7.3A]). Thus, by [Sei83], it suffices to assume that $p \geq 5$. Moreover,

$$
|G| /\left|C_{G}(x)\right|^{2} \geq \frac{q^{36}\left(q^{12}-1\right)\left(q^{9}+1\right)\left(q^{8}-1\right)\left(q^{6}-1\right)\left(q^{5}+1\right)\left(q^{2}-1\right)}{3(q+1)^{12}},
$$

which is at least $q^{55}$ for $q \geq 2$. The possible maximal subgroups containing $x$ are given in Table 10 Again, the hypothesis of Lemma 5 holds and $(x, G)$ cannot be a minimal counterexample.

\section{4. $F_{4}(q)$}

Observe that Inndiag $\left(G_{0}\right)=G_{0}$. If $x$ is unipotent, then $q=3$, and Law95. and Sho74] contain representatives for the classes of elements of order 3. They are listed in Table 11 together with subsystem overgroups of $x$ that show that $(x, G)$ 
TABle 9. Bounds on $\left|x^{G} \cap X_{i}\right|$ for $X_{i}$ a subgroup of $E_{8}(q), p \geq 5$. $d=(2, q-1), e=(3, q-\epsilon), f=(9, q-\epsilon) / e, g=(5, q-\epsilon)$

\begin{tabular}{|c|c|}
\hline$X_{i} \leq E_{8}(q)$ & Bound on $\left|x^{G} \cap X_{i}\right|$ \\
\hline$d . P \Omega^{+}(16, q) \cdot d$ & 0 \\
\hline d. $\left(L(2, q) \times E_{7}(q)\right) . d$ & 0 \\
\hline$f .\left(L^{\epsilon}(9, q)\right) \cdot e \cdot 2, \epsilon=+1$ & 0 \\
\hline$f .\left(L^{\epsilon}(9, q)\right) \cdot e \cdot 2, \epsilon=-1$ & 0 \\
\hline e. $\left(L^{\epsilon}(3, q) \times E_{6}^{\epsilon}(q)\right) \cdot e \cdot 2, \epsilon=+1$ & 0 \\
\hline e. $\left(L^{\epsilon}(3, q) \times E_{6}^{\epsilon}(q)\right) \cdot e \cdot 2, \epsilon=-1$ & 0 \\
\hline$g \cdot\left(L^{\epsilon}(5, q)\right)^{2} \cdot g \cdot 4, \epsilon=+1$ & 5 \\
\hline$g \cdot\left(L^{\epsilon}(5, q)\right)^{2} \cdot g \cdot 4, \epsilon=-1$ & 5 \\
\hline$S U\left(5, q^{2}\right) .4$ & 0 \\
\hline$P G U\left(5, q^{2}\right) .4$ & 0 \\
\hline$d^{2} \cdot\left(P \Omega^{+}(8, q)\right)^{2} \cdot d^{2} \cdot\left(S_{3} \times 2\right)$ & 0 \\
\hline$d^{2} \cdot\left(P \Omega^{+}\left(8, q^{2}\right)\right) \cdot\left(S_{3} \times 2\right)$ & 0 \\
\hline$\left({ }^{3} D_{4}(q)\right)^{2} .6$ & 0 \\
\hline$\left({ }^{3} D_{4}\left(q^{2}\right)\right) \cdot 6$ & 0 \\
\hline$e^{2} \cdot L^{\epsilon}(3, q)^{4} \cdot e^{2} \cdot G L(2,3), \epsilon=+1$ & 0 \\
\hline$e^{2} \cdot L^{\epsilon}(3, q)^{4} \cdot e^{2} \cdot G L(2,3), \epsilon=-1$ & 0 \\
\hline$U\left(3, q^{2}\right)^{2} .8$ & 0 \\
\hline$U\left(3, q^{4}\right) .8$ & 0 \\
\hline$d^{4} \cdot L(2, q)^{8} \cdot d^{4} \cdot A G L(3,2), q>2$ & 0 \\
\hline$(q-1)^{8} \cdot W\left(E_{8}\right)$ & $q^{46}$ \\
\hline$(q+1)^{8} \cdot W\left(E_{8}\right)$ & $q^{46}$ \\
\hline$\left(q^{4}+q^{3}+q^{2}+q+1\right)^{2} \cdot(5 \times S L(2,5))$ & $5 q^{10}$ \\
\hline $\begin{array}{l}\left(q^{2}+q+1\right)^{4} \cdot 2 .(3 \times U(4,2)) \\
\left(a^{2}+1\right)^{4} \cdot\left(4 \circ 2^{1+4}\right)\end{array}$ & $q^{15}$ \\
\hline $\begin{array}{l}\left(q^{2}+1\right)^{4} \cdot\left(402^{14}\right) \cdot A_{6} \cdot 2 \\
q^{8}+q^{7}-q^{5}-q^{4}-q^{3}+q+1 . \mathbb{Z}_{30}\end{array}$ & $\begin{array}{l}q^{2} \\
q^{15}\end{array}$ \\
\hline$\left(q^{4}-q^{2}+1\right)^{2} \cdot\left(\mathbb{Z}_{12} \circ G L(2,3)\right)$ & $q^{10}$ \\
\hline$\left(q^{8}-q^{7}+q^{5}-q^{4}+q^{3}-q+1\right) \cdot \mathbb{Z}_{30}$ & $q^{15}$ \\
\hline$\left(q^{4}-q^{3}+q^{2}-q+1\right)^{2} \cdot(5 \times S L(2,5))$ & $5 q^{10}$ \\
\hline$\left(q^{2}-q+1\right)^{4} \cdot 2 .(3 \times U(4,2))$ & $q^{12}$ \\
\hline $2^{5+10} . S L(5,2)$ (exotic) & 0 \\
\hline $5^{3} . S L(3,5)$ (exotic) & $q^{7}$ \\
\hline
\end{tabular}

cannot be a minimal counterexample. If $x$ is semisimple and contained in a parabolic subgroup, then Lemma 6 implies that $x$ acts non-trivially on all of the components of the Levi complement of some parabolic subgroup $P$. If $P$ is an endnode parabolic subgroup, then the Levi complement is of type $B_{3}(q)$ or $C_{3}(q)$. If $P$ is not an end-node parabolic subgroup, then $P$ is of type $A_{1}(q) A_{2}(q)$. It suffices to assume that $x$ does not centralize the $A_{1}(q)$ or $A_{2}(q)$ components, so $(x, G)$ cannot be a minimal counterexample. Now suppose that $x$ does not lie in any parabolic subgroups. Then $\left|C_{G}(x)\right| \leq(q+1)^{4}$ by [Sei83. However, by [GLS98, Table 4.7.3A], this condition implies that $p \neq 3$. So suppose that $p \geq 5$ and note that

$$
|G| /\left|C_{G}(x)\right|^{2} \geq q^{24}\left(q^{12}-1\right)\left(q^{8}-1\right)\left(q^{6}-1\right)\left(q^{2}-1\right) /(q+1)^{8},
$$


TABLE 10. Bounds on $\left|x^{G} \cap X_{i}\right|$ for subgroups $X_{i}$ of ${ }^{2} E_{6}(q), p \geq 5$. $d=(2, q-1), e=(3, q+1), f=(3, q-1), g=\left(3, q^{2}-1\right)$, $h=(4, q+1)$

\begin{tabular}{|l|l|l|}
\hline$X_{i}$ & Bound on $\left|x^{G} \cap X_{i}\right|$ & Cruder Bound \\
\hline$d .(L(2, q) \times U(6, q) \cdot d e$ & 0 & \\
$e .\left(U(3, q)^{3} \cdot e^{2} \cdot S_{3}\right.$ & 0 & 0 \\
$f . L\left(3, q^{2}\right) \times L(3, q) \cdot g \cdot 2$ & 0 & \\
$U\left(3, q^{3}\right) \cdot(e \times 3)$ & 0 & \\
$d^{2} \cdot\left(P \Omega^{+}(8, q) \times(q+1 / d)^{2}\right) \cdot d^{2} \cdot S_{3}$ & $(q+1)^{2}$ & \\
$\left({ }^{3} D_{4}(q) \times\left(q^{2}-q+1\right)\right) \cdot 3$ & $\left(q^{2}-q+1\right)$ & \\
$h \cdot\left(P \Omega^{-}(10, q) \times(q+1 / h)\right) \cdot h$ & $(q+1)$ & $q^{2}$ \\
$(q+1)^{6} \cdot W\left(E_{6}\right), q \geq 5$ & $(q+1)^{6} .51840$ & $q^{14}$ \\
$\left(q^{2}-q+1\right)^{3} \cdot 3^{1+2} S L(2,3)$ & $\left(q^{2}-q+1\right)^{3}$ & $q^{6}$ \\
$3^{3+3} \cdot S L(3,3)$ & 0 & \\
\hline
\end{tabular}

TABLE 11. Conjugacy class representatives in $F_{4}(3)$

\begin{tabular}{|l|l|l|}
\hline Representative in $F_{4}(3)$ & $\begin{array}{l}\text { Roots generating } \\
\text { subsystem }\end{array}$ & $\begin{array}{l}\text { Subsystem } \\
\text { type }\end{array}$ \\
\hline$x_{1}=x_{1+2}(1) \frac{a}{}$ & $1,1+3$ & $A_{2}(3)$ \\
$x_{2}=x_{1-2}(1) x_{1+2}(-1)$ & $1-2,2-3,3-4,4$ & $B_{4}(3)$ \\
$x_{3}=x_{1-2}(1) x_{1+2}(-\eta)$ & $1-2,2-3,3-4,4$ & $B_{4}(3)$ \\
$x_{4}=x_{2}(1) x_{3+4}(1)$ & $2-3,3-4,4$ & $B_{3}(3)$ \\
$x_{5}=x_{2-3}(1) x_{4}(1) x_{2+3}(1)$ & $2-3,3-4,4$ & $B_{3}(3)$ \\
$x_{6}=x_{2-3}(1) x_{4}(1) x_{2+3}(\eta)$ & $2-3,3-4,4$ & $B_{3}(3)$ \\
$x_{7}=x_{2}(1) x_{1-2+3+4}(1)$ & $2,1-2+3+4$ & $A_{2}(3)$ \\
$x_{8}=x_{2-3}(1) x_{4}(1) x_{1-2}(1)$ & $2-3,1-2,4$ & $A_{2}(3) A_{1}(3)$ \\
$x_{11}=x_{2+3}(1) x_{1+2-3-4}(1) x_{1-2+3+4}(1)$ & $2+3,1+2-3-4$, & $A_{1}(3) A_{2}(3)$ \\
& $1-2+3+4$ & \\
\hline
\end{tabular}

${ }^{a}$ In this case, $x$ is a long root element in $A_{2}(3)$ and so there exist $g_{1}, g_{2}$ such that $\left\langle x, x^{g_{1}}, x^{g_{2}}\right\rangle$ is not solvable.

which is at least $q^{38}$ for $q \geq 2$. It is clear from Table 12 that $(x, G)$ cannot be a minimal counterexample in this case either.

\section{5. ${ }^{2} F_{4}\left(2^{a}\right)^{\prime}$, WHERE $a$ IS ODD}

Suppose that $a>1$. Since $p \neq 2, x$ is semisimple. If $x$ is contained in a parabolic subgroup, then Lemma 6 can be applied. If the resulting subgroup is an end-node parabolic subgroup, then it will be of type ${ }^{2} B_{2}\left(2^{a}\right)$, in which case $(x, G)$ cannot be a minimal counterexample. If $P$ is not an end-node parabolic subgroup, then the Levi complement will be of type $A_{1}\left(2^{2 a}\right)$, so $(x, G)$ cannot be a minimal counterexample in this case either. So suppose that $x$ is not contained in any parabolic subgroups. Then $p \geq 5$, by the same argument as for $F_{4}(q)$, and

$$
|G| /\left|C_{G}(x)\right|^{2} \geq q^{12}\left(q^{6}+1\right)\left(q^{4}-1\right)\left(q^{3}+1\right)(q-1) / 2(q+1)^{8} .
$$


TABLE 12. Bounds on $\left|x^{G} \cap X_{i}\right|$ for subgroups $X_{i}$ of $F_{4}(q), p \geq 5$. $d=(2, q-1), e=(3, q-\epsilon)$

\begin{tabular}{|l|l|}
\hline Type of $X_{i}$ in $G$ & Bound on $\left|x^{G} \cap X_{i}\right|$ \\
with $G_{0}=F_{4}(q)$ & \\
\hline $2 .(L(2, q) \times P S p(6, q)) .2, q$ odd & 0 \\
$d . \Omega(9, q),\left(2, q_{0}\right)$ classes & 0 \\
$d^{2} \cdot P \Omega^{+}(8, q) \cdot S_{3},\left(2, q_{0}\right)$ classes & 0 \\
$3^{3} D_{4}(q) .3(2, p)$ classes & 0 \\
$e .\left(L^{\epsilon}(3, q) \times L^{\epsilon}(3, q)\right) . e \cdot 2, \epsilon=+1$ & $e^{2}$ \\
$e .\left(L^{\epsilon}(3, q) \times L^{\epsilon}(3, q)\right) \cdot e \cdot 2, \epsilon=-1$ & $e^{2}$ \\
$(S p(4, q) \times S p(4, q)) .2$ & 0 \\
$S p\left(4, q^{2}\right) .2$ & 0 \\
$(q-1)^{4} \cdot W\left(F_{4}\right), q=2^{a}, a>2$ & $q^{8}$ \\
$(q+1)^{4} \cdot W\left(F_{4}\right), q=2^{a}, a>1$ & $q^{11}$ \\
$\left(q^{2}+q+1\right)^{2} \cdot(3 \times S L(2,3)), q=2^{a}$ & $q^{6}$ \\
$\left(q^{2}-q+1\right)^{2} \cdot(3 \times S L(2,3)), q=2^{a}, a>1$ & $q^{6}$ \\
$\left(q^{2}+1\right)^{2} \cdot\left(\mathbb{Z}_{30} \circ G L(2,3)\right), q=2^{a}, a>1$ & $q^{9}$ \\
$\left(q^{4}-q^{2}+1\right) \cdot \mathbb{Z}_{30}, q=2^{a}, a>1$ & $q^{7}$ \\
$3^{3} \cdot S L(3,3), q_{0} \geq 5$ & 0 \\
\hline
\end{tabular}

TABLE 13. Bounds on $\left|x^{G} \cap X_{i}\right|$ for subgroups $X_{i}$ of ${ }^{2} F_{4}(q), p \geq 5$. $d=(2, q-1), e=(3, q-\epsilon)$

\begin{tabular}{|l|l|}
\hline Type of $X_{i}$ in $G$ with $G_{0}={ }^{2} F_{4}(q)$ & Bound on $\left|x^{G} \cap X_{i}\right|$ \\
\hline$S U(3, q) \cdot 2$ & 0 \\
$P G U(3, q) .2$ & 0 \\
$\left({ }^{2} B_{2}(q) \times{ }^{2} B_{2}(q)\right) .2$ & 0 \\
$S p(4, q) .2$ & 0 \\
$B_{2}(q): 2$ & 0 \\
${ }^{2} F_{4}\left(q_{0}\right)$ & 0 \\
$(q+1)^{2} \cdot G L(2,3)$ & $q^{4}$ \\
$(q+\sqrt{2 q}+1)^{2} \cdot\left(\mathbb{Z}_{4} \circ G L(2,3)\right)$ & $q^{4}$ \\
$(q-\sqrt{2 q}+1)^{2} \cdot\left(\mathbb{Z}_{4} \circ G L(2,3)\right), q>8$ & $q^{2}$ \\
$\left(q^{2}+\sqrt{2 q^{3}}+q+\sqrt{2 q}+1\right) \cdot \mathbb{Z}_{12}$ & $q^{5}$ \\
$\left(q^{2}-\sqrt{2 q^{3}}+q-\sqrt{2 q}+1\right) \cdot \mathbb{Z}_{12}$ & $q^{2}$ \\
\hline
\end{tabular}

This is at least $q^{15}$ for $q \geq 8$. The maximal subgroups are given in Mal91 and include the calculations in Table 13 .

Thus $(x, G)$ cannot be a minimal counterexample for $q \geq 8$. If $a=1$, then $q=2$ and the possibilities for the order of $x$ are 3,5 , and 13. There are unique classes of cyclic subgroups of orders 3,5 , and 13, by [CCN85]; thus a conjugate of $x$ is contained in a subgroup isomorphic to $\operatorname{PSL}(2,25)$. So $(x, G)$ cannot be a minimal counterexample in this case either.

The only outer automorphisms are field automorphisms. If $x$ is a field automorphism, then $x$ normalizes an end-node parabolic subgroup and acts non-trivially on the Levi complement. Therefore, $(x, G)$ cannot be a minimal counterexample. 


\section{6. $G_{2}(q)$}

Observe that, since $G_{2}(2)^{\prime} \cong P S U(3,3)$, it suffices to assume that $q \neq 2$. First consider the case where $q$ is a power of 2 ; so in particular, $x$ is semisimple. The algebraic group $G_{2}$ fixes a non-degenerate quadratic form by [SS97, 4.1] and [LSS96] for example. It follows that any element of $G_{0}$ is conjugate to an element of either $S L_{3}(q): 2$ or $S U(3, q): 2$. Either $x$ is non-central in one of these groups, in which case $(x, G)$ is not a minimal counterexample, or $x$ is central in $S L^{\epsilon}(3, q)$, and therefore is contained in a parabolic subgroup $P$. In the latter case, applying Lemma 6 implies that it suffices to assume that $x$ acts non-centrally on the Levi complement, which is of type $A_{1}(q)$. So $(x, G)$ is not a minimal counterexample in this case either.

Now suppose that $q$ is odd. If $x$ is semisimple, then, since it has odd order, it must be contained in $S L^{\epsilon}(3, q)$ for either $\epsilon=+$ or $\epsilon=-$. Thus if $x$ is not a central element in this subgroup, then $(x, G)$ is not a minimal counterexample. If $x$ is central in the $S L^{\epsilon}(3, q)$, then $x$ is contained in a parabolic subgroup $P$. So by Lemma 6, it suffices to assume that $x$ acts non-centrally on the Levi complement, which is of type $A_{1}(q)$. Since $p \mid q-\epsilon$ and $q$ is odd, it follows that $q \geq 5$ and $(x, G)$ cannot be a minimal counterexample in this case either. Similarly, if $x$ is unipotent and $q \neq 3$, then Lemma 6 implies that $x$ acts non-trivially on an $A_{1}(q)$ Levi component of a parabolic subgroup.

Suppose that $q=3=p$ and that $x$ is not a root element. It is easily verified using MAGMA that there are two conjugacy classes of elements of order 3 (long root elements and short root elements) that belong in Table 1. Moreover, in these cases, there exist $g_{1}, g_{2} \in G_{2}(3)$ such that $\left\langle x, x^{g_{1}}, x^{g_{2}}\right\rangle$ is not solvable.

$$
\text { 17. }{ }^{3} D_{4}(q)
$$

One can use MAGMA for the cases $q=2$ and $q=3$, so assume from now on that $q \geq 4$. If $x$ is unipotent, then, by Lemma 6 it suffices to assume that $x$ acts non-centrally on a Levi component of a parabolic subgroup of type $A_{1}(q)$, or $A_{1}\left(q^{3}\right)$. So, since $q \geq 4,(x, G)$ cannot be a minimal counterexample. Similarly, if $x$ is semisimple and is contained in a parabolic subgroup, then Lemma 6 applies, as in the unipotent case. So it suffices to assume that $x$ is not contained in any parabolic subgroups. It follows that $C_{G}(x)$ is a torus and $\left|C_{G}(x)\right| \leq(q+1)^{4}$ by Sei83. Thus,

$$
\frac{|G|}{\left|C_{G}(x)\right|^{2}} \geq \frac{q^{12}\left(q^{8}+q^{4}+1\right)\left(q^{6}-1\right)\left(q^{2}-1\right)}{(q+1)^{8}},
$$

which is at least $q^{18}$ for $q \geq 4$. As usual, observe that $p \geq 5$ by [GLS98. The possible maximal subgroups containing $x$ can be deduced from [Kle88b] and are listed in Table 14 and Lemma 5 shows that $(x, G)$ cannot be a minimal counterexample.

$$
\text { 18. }{ }^{2} B_{2}\left(2^{a}\right), a \neq 1 \text { ODD }
$$

If $a=1$, then ${ }^{2} B_{2}\left(2^{a}\right)$ is solvable, so it suffices to assume that $a \neq 1$. The maximal subgroups are described in [Suz62] and are listed in Table 15] Note that $|G|=q^{2}\left(q^{2}+1\right)(q-1)$, where $q=2^{a}$. Also, observe that $p \nmid q$ since $p$ is odd and it suffices to assume that the only subfield subgroup that can contain $x$ is ${ }^{2} B_{2}(2)$, since otherwise $(x, G)$ would not be a minimal counterexample. By Suz62, Theorem 4], for example, any element of odd order in ${ }^{2} B_{2}(q)$ has its centralizer 
TABLE 14. Bounds on $\left|x^{G} \cap X_{i}\right|$ for subgroups $X_{i}$ of ${ }^{3} D_{4}(q), q \geq 4$, and $p \geq 5 . d=(2, q-1), e=(3, q-\epsilon), f=\left(3, q^{2}+\epsilon q+1\right)$

\begin{tabular}{|l|l|}
\hline Type of $X_{i}$ in $G$ with $G_{0}={ }^{3} D_{4}(q)$ & Bound on $\left|x^{G} \cap X_{i}\right|$ \\
\hline$G_{2}(q)$ & 0 \\
$P G L^{\epsilon}(3, q), q \equiv \epsilon(\bmod 3)$ & 0 \\
${ }^{3} D_{4}\left(q_{1}\right), q_{1}^{\alpha}=q, \alpha \neq 3$ prime & 0 \\
$L\left(2, q^{3}\right) \times L(2, q), q_{0}=2$ & 0 \\
$\left(S L\left(2, q^{3}\right) \circ S L(2, q)\right) .2, q_{0} \neq 2$ & 0 \\
$\left(\left(q^{2}+q+1\right) \circ S L(3, q)\right) \cdot\left(3, q^{2}+q+1\right) .2$ & $q^{3}$ \\
$\left(\left(q^{2}-q+1\right) \circ S U(3, q)\right) \cdot\left(3, q^{2}-q+1\right) .2$ & $q^{2}$ \\
$\left(q^{2}+q+1\right)^{2} \cdot S L(2,3)$ & $(q+1)^{4}$ \\
$\left(q^{2}-q+1\right)^{2} \cdot S L(2,3)$ & $q^{4}$ \\
$\left(q^{4}-q^{2}+1\right) \cdot 4$ & $q^{4}$ \\
\hline
\end{tabular}

TABLE 15. Maximal subgroups of ${ }^{2} B_{2}\left(2^{a}\right)$

\begin{tabular}{|l|l|l|}
\hline Subgroup & Bound on $\left|x^{G} \cap M\right|$ & Comments \\
\hline$H$ & $q^{2}(q-1)$ & Borel subgroup \\
$D_{2(q-1)}$ & $2(q-1)$ & maximal rank \\
$N\left(A_{1}\right)$ & $4(q+\sqrt{2 q}+1)$ & maximal rank \\
$N\left(A_{2}\right)$ & $4(q-\sqrt{2 q}+1)$ & maximal rank \\
${ }^{2} B_{2}\left(2^{a / b}\right), b \mid a$, & $q^{2 / b}\left(q^{2 / b}+1\right)\left(q^{1 / b}-1\right)$ & One class [Suz62, Theorem 10] \\
\hline
\end{tabular}

contained in one of the cyclic groups of order $q-1, q+\sqrt{2 q}+1$ or $q-\sqrt{2 q}+1$. So there are three mutually exclusive possibilities for $p: p|q-1, p| q+\sqrt{2 q}+1$, and $p \mid q-\sqrt{2 q}+1$. If $p \mid q-1$, then

$$
|G| /\left|C_{G}(x)\right|^{2} \geq q^{2}\left(q^{2}+1\right)(q-1) /(q-1)^{2}
$$

and

$$
\sum_{i}\left|x^{G} \cap X_{i}\right| \leq q^{2}(q-1)+2(q-1)+\left.\right|^{2} B_{2}(2) \mid .
$$

An elementary calculation shows that since $q \geq 8$,

$$
|G| /\left|C_{G}(x)\right|^{2}>\sum_{i}\left|x^{G} \cap X_{i}\right|
$$

and Lemma 5 applies. Similarly if $p \mid q \pm \sqrt{2 q}+1$, then

$$
\sum_{i}\left|x^{G} \cap X_{i}\right| \leq 4\left(q^{2} \pm \sqrt{2 q}+1\right)+\left|{ }^{2} B_{2}(2)\right|
$$

and the hypotheses of Lemma 5 are satisfied. Thus, $(x, G)$ cannot be a minimal counterexample.

If $x$ is an outer automorphism, then it must be a field automorphism and the same counting argument as for $P S L(2, q)$ applies. Observe that it suffices to assume that there are no subfield subgroups among the $H_{i}$ 's except ${ }^{2} B_{2}\left(q_{0}\right)=C_{G_{0}}(x)$. If there were, then $x$ would be contained in $\operatorname{Aut}\left({ }^{2} B_{2}\left(q^{1 / r}\right)\right)$ for some prime $r \neq p$ and $(x, G)$ would not be a minimal counterexample. So

$$
\left|G_{0}\right| /\left|C_{G_{0}}(x)\right|^{2}=q^{2}\left(q^{2}+1\right)(q-1) / q_{0}^{4}\left(q_{0}^{2}+1\right)^{2}\left(q_{0}-1\right)^{2}
$$


TABLE 16. Maximal subgroups of ${ }^{2} G_{2}\left(3^{a}\right)$

\begin{tabular}{|l|l|}
\hline Subgroup & Comments \\
\hline$P=\left[q^{3}\right] \cdot(q-1)$ & Borel subgroup, only one class \\
$2 \times L(2, q), q \geq 27$ & maximal rank \\
$\left(2^{2} \times D_{(q+1) / 2}\right): 3, q \geq 27$ & maximal rank \\
$\mathbb{Z}_{q+\sqrt{3 q}+1}: \mathbb{Z}_{6}$ & maximal rank \\
$\mathbb{Z}_{q-\sqrt{3 q}+1}: \mathbb{Z}_{6}, q \geq 27$ & maximal rank \\
${ }^{2} G_{2}\left(q_{0}\right), q=q_{0}^{\alpha}, \alpha$ prime & \\
\hline
\end{tabular}

and

$$
\begin{aligned}
1+\sum_{i=1}^{m} \frac{\left|H_{i}\right|}{\left|C_{H_{i}}(x)\right|^{2}} \leq & 1+\frac{q^{2}(q-1)}{q_{0}^{4}\left(q_{0}-1\right)^{2}}+\frac{2(q-1)}{\left(q_{0}-1\right)^{2}} \\
& +\frac{4(q+\sqrt{2 q}+1)}{\left(q_{0}+\sqrt{2 q_{0}}+1\right)^{2}}+\frac{4(q-\sqrt{2 q}+1)}{\left(q_{0}-\sqrt{2 q_{0}}+1\right)^{2}} .
\end{aligned}
$$

A computation shows that the required inequality holds for all $q \geq 2^{3}$ and all $p \geq 3$.

$$
\text { 19. }{ }^{2} G_{2}\left(3^{a}\right), a \neq 1 \text { ODD }
$$

Observe that if $a=1$, then ${ }^{2} G_{2}^{\prime}(3) \cong L(2,8)$, so suppose that $a \neq 1$. Also, $|G|=q^{3}\left(q^{3}+1\right)(q-1)$ and the maximal subgroups are given in Kle88a, which are listed in Table 16. If $p \nmid q=3^{a}$, then there are three mutually exclusive possibilities: $p\left|\left(q^{2}-1\right), p\right| q-\sqrt{3 q}+1$, and $p \mid q+\sqrt{3 q}+1$. First suppose that $p \mid q^{2}-1$. Then a Sylow $p$-subgroup is contained inside a maximal subgroup $2 \times P S L(2, q)$, so some conjugate of $x$ is contained in $\operatorname{PSL}(2, q)$. Thus, $(x, G)$ cannot be a minimal counterexample.

If $p \mid q^{2}-q+1$, then a Sylow $p$-subgroup is contained in one of the abelian Hall subgroups of order $q \pm \sqrt{3 q}+1$, so it suffices to assume that $x$ is contained in one of these Hall subgroups and that $\left|C_{G}(x)\right|=q \pm \sqrt{3 q}+1$ (see part (4) of the main theorem in [War66]). Then an easy count shows that the hypotheses of Lemma 5 are satisfied. If $p \mid q$, then War66 shows that there are three conjugacy classes of elements of order $p=3$. One class contains elements in the center of a Sylow 3-subgroup and these elements have centralizers of order $q^{3}$. The other two conjugacy classes have centralizers of order $2 q^{2}$. Elements in these classes centralize an involution $w$, so they are contained in $C_{G}(w) \cong L(2, q) \times 2$ and so $(x, G)$ cannot be a minimal counterexample in this case. Now Law95 gives a representative $x_{2 a+b}(1) x_{3 a+2 b}(1)$ for the conjugacy class of elements $t$ with $\left|C_{G}(t)\right|=q^{3}$. This is contained in ${ }^{2} G_{2}(3) \cong L(2,8): 3$, so $(x, G)$ cannot be a minimal counterexample in this case either. If $x$ is an outer automorphism, then it must be a field automorphism. The same method as for ${ }^{2} B_{2}\left(2^{a}\right)$ applies here. As before, it suffices to assume that there are no subfield subgroups among the $H_{i}$ 's, other than ${ }^{2} B_{2}\left(2^{a / p}\right)$. So

$$
\left|G_{0}\right| /\left|C_{G_{0}}(x)\right|^{2}=q^{3}\left(q^{3}+1\right)(q-1) / q_{0}^{6}\left(q_{0}^{3}+1\right)^{2}\left(q_{0}-1\right)^{2}
$$


and

$$
\begin{aligned}
1+\sum_{i=1}^{m} \frac{\left|H_{i}\right|}{\left|C_{H_{i}}(x)\right|^{2}} \leq & 1+\frac{q^{3}(q-1)}{q_{0}^{6}\left(q_{0}-1\right)^{2}}+\frac{6(q+1)}{\left(q_{0}+1\right)^{2}}+\frac{6(q+\sqrt{3 q}+1)}{\left(q_{0}+\sqrt{3 q_{0}}+1\right)^{2}} \\
& +\frac{6(q-\sqrt{3 q}+1)}{\left(q_{0}-\sqrt{3 q_{0}}+1\right)^{2}}+\frac{2 q\left(q^{2}-1\right)}{q_{0}^{2}\left(q_{0}^{2}-1\right)^{2}} .
\end{aligned}
$$

A computation now shows that $(x, G)$ cannot be a minimal counterexample for any prime power $q$.

\section{SporadiC GROUPS}

If $G_{0}$ is one of the following sporadic groups, then a MAGMA calculation shows that there exists $g \in G$ such that $\left\langle x, x^{g}\right\rangle$ is not solvable:

$$
\begin{aligned}
& M_{11}, M_{12}, M_{22}, M_{23}, M_{24}, J_{1}, J_{2}, J_{3}, C o_{2}, \\
& C o_{3}, M c L, H S, S u z, H e, F i_{22}, F i_{23}, F i_{24} .
\end{aligned}
$$

There are 9 remaining sporadic groups, which are a little more awkward. One can use CCN85, which describes the conjugacy classes and maximal subgroups. In certain circumstances, one can show that some element of a conjugacy class is contained inside some smaller almost simple group. In particular, one can do this if there is a unique conjugacy class of elements of order $p$, or a multiple of $p$ that powers up to the conjugacy class in question. Then any almost simple subgroup containing elements of this order will contain an element of $x^{G}$, and thus $(x, G)$ cannot be a minimal counterexample. Clearly, this also applies if all of the conjugacy classes of elements of order $p$ are powers of each other. In the remaining cases, one can use MAGMA with a little more care. The details are listed in Tables $17,18,19,20,21,22,23,24$, and 25,

This completes the proof of Theorem A*. 
TABLE 17. Janko group, $J_{4}$

\begin{tabular}{|c|c|c|}
\hline Class(es) & MAGMA & $\begin{array}{c}\text { Subgroup containing } x \\
\text { and class that powers up to } x\end{array}$ \\
\hline 3 & & $M_{22}: 2,3$ \\
5 & & $M_{22}: 2,5$ \\
7 & & $M_{22}: 2,7 \frac{a}{}$ \\
$11 \mathrm{~A}$ & & $P S U(3,11): 2,44$ \\
$11 \mathrm{~B}$ & & $\langle x, a\rangle$ generates $^{b}$ \\
23 & $2^{11}: M_{24}, 23$ \\
29 & $\langle x, a\rangle$ generates $^{c}$ \\
31 & $L(2,32), 31$ \\
37 & $U(3,11), 37$ \\
43 & & $\langle x, a\rangle$ generates $^{d}$ \\
\hline
\end{tabular}

${ }^{a} 7 A=(7 B)^{3}, 7 B=7 A^{3}$.

${ }^{b}$ In this case, $a$ is a standard generator in class $2 \mathrm{~A} ; x$ is a standard representative for class 11B; $x^{3} a$ has order 43 and $x^{2} a$ has order 35 , so $\langle x, a\rangle$ cannot be contained in any maximal subgroups; thus $\left\langle x, x^{a}\right\rangle$ is not solvable since its index in $\langle x, a\rangle$ is at most 2 .

${ }^{c}$ In this case, $x$ is a standard representative for class 29A. We can show in MAGMA that the group order is a multiple of 29.44 .

${ }^{d}$ In this case, $x$ is a standard representative for class $43 \mathrm{~A}$, but a calculation in MAGMA shows that 43.23 divides the order of $\langle x, a\rangle$.

TABle 18. Conway group, $C o_{1}$

\begin{tabular}{|c|c|c|}
\hline Class(es) & MAGMA & $\begin{array}{c}\text { Subgroup containing } x \\
\text { and class that powers up to } x\end{array}$ \\
\hline 3 & done & \\
5 & done & $A_{9}, 42$ \\
$7 \mathrm{~A}$ & & \\
$7 \mathrm{~B}$ & done & $\mathrm{Co}_{3}, 11$ \\
11 & & $3: \mathrm{Suz}_{2}: 2,13$ \\
13 & & $\mathrm{Co}_{2}, 23$ \\
23 & & \\
\hline
\end{tabular}

TABLE 19. Rudvalis group, $R u$

\begin{tabular}{|c|c|c|}
\hline Class(es) & MAGMA & $\begin{array}{c}\text { Subgroup containing } x \\
\text { and class that powers up to } x\end{array}$ \\
\hline 3 & done & \\
5 & done & $A_{8}, 7$ \\
7 & & $P S L(2,13), 13$ \\
13 & & $P S L(2,29), 29$ \\
29 & & $P$ \\
\hline
\end{tabular}


TABle 20. O'Nan group, $O^{\prime} N$

\begin{tabular}{|c|c|c|}
\hline Class(es) & MAGMA & $\begin{array}{c}\text { Subgroup containing } x \\
\text { and class that powers up to } x\end{array}$ \\
\hline 3 & done & $A_{7}, 5$ \\
5 & & $P S L(3,7), 14$ \\
$7 \mathrm{~A}$ & & \\
$7 \mathrm{~B}$ & done & $J_{1}, 11$ \\
11 & & $J_{1}, 19$ \\
19 & & $P S L(2,31), 31$ \\
31 & & $P$ \\
\hline
\end{tabular}

TABLE 21. Harada-Norton group, $H N$

\begin{tabular}{|c|c|c|}
\hline Class(es) & MAGMA & $\begin{array}{c}\text { Subgroup containing } x \\
\text { and class that powers up to } x\end{array}$ \\
\hline 3A & & $A_{12}, 21 \mathrm{~A}$ \\
3B & & $A_{12}, 9$ \\
5A & & $A_{12}, 35$ \\
5B-E & done $^{a}$ & \\
7 & & $A_{12}, 7$ \\
11 & & $A_{12}, 11$ \\
19 & & $P S U(3,8), 19$ \\
\hline
\end{tabular}

${ }^{a}$ MAGMA calculation performed using permutation representation in $\mathrm{ABN}$.

TABLE 22. Lyons group, Ly

\begin{tabular}{|c|c|c|}
\hline Class(es) & MAGMA & $\begin{array}{c}\text { Subgroup containing } x \\
\text { and class that powers up to } x\end{array}$ \\
\hline 3A & & $2 . A_{11}, 21 \mathrm{~A}$ \\
3B & & $2 . A_{11}, 9$ \\
5A & & $2 . A_{11}, 20$ \\
5B & done $^{a}$ & \\
7 & & $2 . A_{11}, 7$ \\
11 & & $2 . A_{11}, 11$ \\
31 & & $5^{3} . P S L(3,5), 31$ \\
37 & done $^{b}$ & \\
67 & done $^{-}$ & \\
\hline
\end{tabular}

${ }^{a}$ If $a, b$, and $x$ are standard representatives for classes $2 \mathrm{~A}, 5 \mathrm{~A}$, and $5 \mathrm{~B}$, respectively, then $a x^{b}$ has order 67 , so $\left\langle a, x^{b}\right\rangle$ cannot be contained in any maximal subgroup.

${ }^{b}$ If $a$ and $x$ are standard representatives for classes $2 \mathrm{~A}$ and $37 \mathrm{~A}$, respectively, then $a x$ has order 67 , so $\langle a, x\rangle$ cannot be contained in any maximal subgroup.

${ }^{c}$ If $a$ and $x$ are standard representatives for classes $2 \mathrm{~A}$ and $67 \mathrm{~A}$, respectively, then $a x$ has order 14 , so $\langle a, x\rangle$ cannot be contained in any maximal subgroup. 
TABle 23. Thompson Group, $T h$

\begin{tabular}{|c|c|c|}
\hline Class(es) & MAGMA & $\begin{array}{c}\text { Subgroup containing } x \\
\text { and class that powers up to } x\end{array}$ \\
\hline 3A & & $P S U(3,8), 21$ \\
3B & & $A_{9}, 9$ \\
3C & & $A_{9}, 15$ \\
5 & & $A_{9}, 5$ \\
7 & & $A_{9}, 7$ \\
13 & & $P S L(3,3), 13$ \\
19 & & $P S L(2,19), 19$ \\
31 & & $2^{5} . P S L(5,2), 31$ \\
\hline
\end{tabular}

TABLE 24. Baby Monster, $B$

\begin{tabular}{|c|c|c|}
\hline Class(es) & MAGMA & $\begin{array}{c}\text { Subgroup containing } x \\
\text { and class that powers up to } x\end{array}$ \\
\hline 3A & & $H N, 21$ \\
3B & & $H N, 9$ \\
5A & & $H N, 35$ \\
5B & & $H N, a$ \\
7 & & $P S L(2,49), 7$ \\
11 & & $P S L(2,11), 11$ \\
13 & & $P S L(3,3), 13$ \\
17 & & $P S L(2,17), 17$ \\
19 & & $H N, 19$ \\
23 & & $F I_{23}, 23$ \\
31 & & $P S L(2,31), 31$ \\
47 & done $\underline{\underline{-}}$ & \\
\hline
\end{tabular}

${ }^{a}$ The order of $C_{B}(x)$ is a multiple of $5^{6}$, but $5^{5} \nmid C_{B}(y)$ if $y \in 5 A$, so any member of the class $5 \mathrm{~B}$ in $H N$ (centralizer order 500,000) must be in the Baby Monster class $5 \mathrm{~B}$.

${ }^{b}$ Since $a x$ has order 9, where $a$ and $x$ are standard representatives for classes $2 \mathrm{~A}$ and $47 \mathrm{~A}$, respectively, $\langle a, x\rangle$ generates since the only maximal subgroup with order a multiple of 47 is $47: 23$. 
SIMON GUEST

TABle 25. Monster Group, $M$

\begin{tabular}{|c|c|c|}
\hline Class(es) & $\left|C_{G}(x)\right|$ & $\begin{array}{l}\text { Subgroup containing } x \\
\text { and class that powers up to } x\end{array}$ \\
\hline $3 \mathrm{~A}$ & & $B, 48$ \\
\hline $3 \mathrm{~B}$ & & $A_{12}, 9$ \\
\hline $3 \mathrm{C}$ & & $P S U(3,8) \times A_{5}, 57$ \\
\hline $5 \mathrm{~A}$ & & $P S L(2,11) \times M_{12}, 55$ \\
\hline $5 \mathrm{~B}$ & & $P S L(2,25), 25$ \\
\hline $7 \mathrm{~A}$ & & $\left(A_{5} \times A_{12}\right), 105$ \\
\hline $7 \mathrm{~B}$ & & contained in $\operatorname{PSL}(2,71)$ group by [NW02, pg 596] \\
\hline 11 & & $2 . B, 11$ \\
\hline $13 \mathrm{~A}$ & 73008 & $S_{3} \times T h^{a}$ \\
\hline 13B & 52728 & Lies in 6.Suz by [NW02, pg 593] \\
\hline 17 & & $2 . B, 17$ \\
\hline 19 & & 2.B, 19 \\
\hline 23 & & 2. $B, 23$ \\
\hline 29 & & 3. $F i_{24}, 29$ \\
\hline 31 & & 2. $B, 31$ \\
\hline 41 & & $3^{8} . O^{-}(8,3), 41$ \\
\hline 47 & & 2. $B, 47$ \\
\hline 59 & & $P S L(2,59), 59$ \\
\hline 71 & & $\operatorname{PSL}(2,71), 71$ \\
\hline
\end{tabular}

${ }^{a}$ Since an element of order 13 in $T h$ has centralizer order 39 , it follows that any such element is in $13 \mathrm{~A}$ since 39.6 divides $\left|C_{G}(x)\right|$ but does not divide 52728 . 


\section{REFERENCES}

[ABN] Rachel Abbott, John Bray, Simon Nickerson, Steve Linton, Simon Norton, Richard Parker, Ibrahim Suleiman, Jonathan Tripp, Peter Walsh, and Robert Wilson, A www-atlas of finite group representations.

[AG84] M. Aschbacher and R. Guralnick, Some applications of the first cohomology group, J. Algebra 90 (1984), no. 2, 446-460. MR760022 (86m:20060)

[Bos] Wieb Bosma, John Cannon, and Catherine Playoust. The Magma algebra system. I. The user language. J. Symbolic Comput. 24 (3-4): 235-265, 1997. MR1484478

[Bur04] Timothy C. Burness, Fixed point spaces in actions of classical algebraic groups, J. Group Theory 7 (2004), no. 3, 311-346. MR2063000 (2005c:14054)

[CCN85] J. H. Conway, R. T. Curtis, S. P. Norton, R. A. Parker, and R. A. Wilson, Atlas of finite groups, Oxford University Press, Eynsham, 1985, Maximal subgroups and ordinary characters for simple groups, With computational assistance from J. G. Thackray. MR827219 (88g:20025)

[Enn62] Veikko Ennola, On the conjugacy classes of the finite unitary groups, Ann. Acad. Sci. Fenn. Ser. A I No. 313 (1962), 13pp. MR.0139651 (25:3082)

[FGG] Paul Flavell, Robert Guralnick, and Simon Guest, Characterizations of the solvable radical, Proc. Amer. Math. Soc. 138 (2010), no. 4, 1161-1170. MR2578510

[GGKP08a] Nikolai Gordeev, Fritz Grunewald, Boris Kunyavskii, and Eugene Plotkin, A commutator description of the solvable radical of a finite group, Groups Geom. Dyn. 2 (2008), no. 1, 85-120. MR2367209 (2008j:20057)

[GGKP08b] _ A description of Baer-Suzuki type of the solvable radical of a finite group, J. Pure and Applied Algebra 213 (2009), 250-258. MR2467402 (2009i:20045)

[GHL96] Meinolf Geck, Gerhard Hiss, Frank Lübeck, Gunter Malle, and Götz Pfeiffer, CHEVIE-a system for computing and processing generic character tables, Appl. Algebra Engrg. Comm. Comput. 7 (1996), no. 3, 175-210, Computational methods in Lie theory (Essen, 1994). MR 1486215 (99m:20017)

[GK00] Robert M. Guralnick and William M. Kantor, Probabilistic generation of finite simple groups, J. Algebra 234 (2000), no. 2, 743-792, Special issue in honor of Helmut Wielandt. MR1800754 (2002f:20038)

[GL83] Daniel Gorenstein and Richard Lyons, The local structure of finite groups of characteristic 2 type, Mem. Amer. Math. Soc. 42 (1983), no. 276, vii+731pp. MR690900 (84g:20025)

[GLS98] Daniel Gorenstein, Richard Lyons, and Ronald Solomon, The classification of the finite simple groups. Number 3, Mathematical Surveys and Monographs, vol. 40, American Mathematical Society, Providence, RI, 1998. MR 1490581 (98j:20011)

[GPPS99] Robert Guralnick, Tim Penttila, Cheryl E. Praeger, and Jan Saxl, Linear groups with orders having certain large prime divisors, Proc. London Math. Soc. (3) 78 (1999), no. 1, 167-214. MR.1658168 (99m:20113)

[GPS07] Robert Guralnick, Eugene Plotkin, and Aner Shalev, Burnside-type problems related to solvability, Internat. J. Algebra Comput. 17 (2007), no. 5-6, 1033-1048. MR2355682 (2008j:20110)

[GS03] Robert M. Guralnick and Jan Saxl, Generation of finite almost simple groups by conjugates, J. Algebra 268 (2003), no. 2, 519-571. MR2009321(2005f:20057)

[Gur98] Robert M. Guralnick, Some applications of subgroup structure to probabilistic generation and covers of curves, Algebraic groups and their representations (Cambridge, 1997), NATO Adv. Sci. Inst. Ser. C Math. Phys. Sci., vol. 517, Kluwer Acad. Publ., Dordrecht, 1998, pp. 301-320. MR.1670777(2000d:20062)

[KL90] Peter Kleidman and Martin Liebeck, The subgroup structure of the finite classical groups, London Mathematical Society Lecture Note Series, vol. 129, Cambridge University Press, Cambridge, 1990. MR.1057341 (91g:20001)

[Kle88a] Peter B. Kleidman, The maximal subgroups of the Chevalley groups $G_{2}(q)$ with $q$ odd, the Ree groups ${ }^{2} G_{2}(q)$, and their automorphism groups, J. Algebra 117 (1988), no. 1, 30-71. MR955589 (89j:20055)

[Kle88b] _ The maximal subgroups of the Steinberg triality groups ${ }^{3} D_{4}(q)$ and of their automorphism groups, J. Algebra 115 (1988), no. 1, 182-199. MR937609 (89f:20024) 
[Law95] R. Lawther, Jordan block sizes of unipotent elements in exceptional algebraic groups, Comm. Algebra 23 (1995), no. 11, 4125-4156. MR.1351124 (96h:20084)

[LS03] Martin W. Liebeck and Gary M. Seitz, A survey of maximal subgroups of exceptional groups of Lie type, Groups, combinatorics \& geometry (Durham, 2001), World Sci. Publ., River Edge, NJ, 2003, pp. 139-146. MR.1994964 (2004f:20089)

[LSS92] Martin W. Liebeck, Jan Saxl, and Gary M. Seitz, Subgroups of maximal rank in finite exceptional groups of Lie type, Proc. London Math. Soc. (3) 65 (1992), no. 2, 297-325. MR1168190 (93e:20026)

[LSS96] _ Factorizations of simple algebraic groups, Trans. Amer. Math. Soc. 348 (1996), no. 2, 799-822. MR 1316858 (96g:20064)

[Mal91] Gunter Malle, The maximal subgroups of ${ }^{2} F_{4}\left(q^{2}\right)$, J. Algebra 139 (1991), no. 1, 52-69. MR 1106340 (92d:20068)

[Miz77] Kenzo Mizuno, The conjugate classes of Chevalley groups of type $E_{6}$, J. Fac. Sci. Univ. Tokyo Sect. IA Math. 24 (1977), no. 3, 525-563. MR0486170 (58:5951)

[Miz80] The conjugate classes of unipotent elements of the Chevalley groups $E_{7}$ and $E_{8}$, Tokyo J. Math. 3 (1980), no. 2, 391-461. MR605099 (82m:20046)

[NW02] Simon P. Norton and Robert A. Wilson, Anatomy of the Monster. II, Proc. London Math. Soc. (3) 84 (2002), no. 3, 581-598. MR1888424 (2003b:20023)

[Sei83] Gary M. Seitz, The root subgroups for maximal tori in finite groups of Lie type, Pacific J. Math. 106 (1983), no. 1, 153-244. MR694680 (84g:20085)

[Sho74] Toshiaki Shoji, The conjugacy classes of Chevalley groups of type $\left(F_{4}\right)$ over finite fields of characteristic $p \neq 2$, J. Fac. Sci. Univ. Tokyo Sect. IA Math. 21 (1974), 1-17. MR0357641 (50:10109)

[SS97] Jan Saxl and Gary M. Seitz, Subgroups of algebraic groups containing regular unipotent elements, J. London Math. Soc. (2) 55 (1997), no. 2, 370-386. MR.1438641 (98m:20057)

[Suz62] Michio Suzuki, On a class of doubly transitive groups, Ann. of Math. (2) 75 (1962), 105-145. MR0136646 (25:112)

[Tho68] John G. Thompson, Nonsolvable finite groups all of whose local subgroups are solvable, Bull. Amer. Math. Soc. 74 (1968), 383-437. MR0230809 (37:6367)

[Wal63] G. E. Wall, On the conjugacy classes of classical groups, J. Austral. Math. Soc. 3 (1963), 1-62. MR0150210 (27:212)

[War66] Harold N. Ward, On Ree's series of simple groups, Trans. Amer. Math. Soc. 121 (1966), 62-89. MR0197587 (33:5752)

[Wie64] Helmut Wielandt, Finite permutation groups, Translated from the German by R. Bercov, Academic Press, New York, 1964. MR0183775 (32:1252)

Department of Mathematics, University of Southern California, Los Angeles, CalIFORNIA 90089-2532

Current address: Department of Mathematics, Baylor University, One Bear Place, \#97328, Waco, Texas 76798

E-mail address: sguest@usc.edu 